Supporting Information for

\title{
Sub-stoichiometric 3D Covalent Organic Frameworks Based on Hexagonal Linkers
}

Liangjun Chen, ${ }^{\dagger}, *$ Chengtao Gong, ${ }^{\dagger}, *$ Xiaokang Wang, ${ }^{\dagger}, *$ Fangna Dai, ${ }^{*}$, Mingchu Huang, $^{\dagger}$ Xiaowei $\mathrm{Wu},{ }^{*,,, \#}$ Can-Zhong Lu, ${ }^{\S, \#, \uparrow}$ and Yongwu Peng ${ }^{*, \dagger}$

${ }^{\dagger}$ College of Materials Science and Engineering and College of Chemical Engineering, Zhejiang University of Technology, Hangzhou 310014, China.

‡School of Materials Science and Engineering, China University of Petroleum (East China), Qingdao 266580, China.

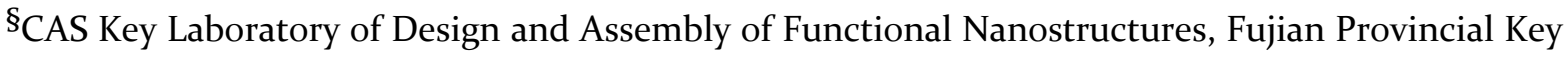
Laboratory of Nanomaterials, Fujian Institute of Research on the Structure of Matter (FJIRSM), Chinese Academy of Sciences, Fuzhou 350002, China.

\#Xiamen Key Laboratory of Rare Earth Photoelectric Functional Materials, Xiamen Institute of Rare Earth Materials (XMIREM), Haixi Institutes, Chinese Academy of Sciences, Xiamen 361021, China.

IUniversity of Chinese Academy of Sciences, Beijing 100049, China.

※These authors contributed equally to this work.

E-mail: yongwupeng@gmail.com (Y.P.); xmwuxiaowei@fjirsm.ac.cn (X. W.);

fndai@upc.edu.cn (F. D.); 


\section{Table of Content}

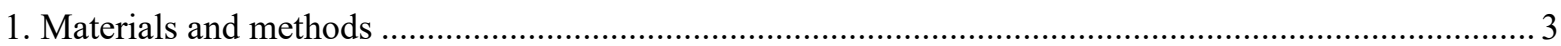

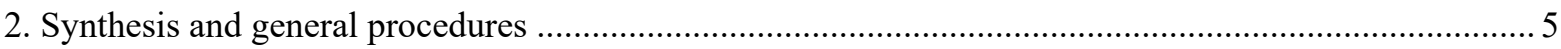

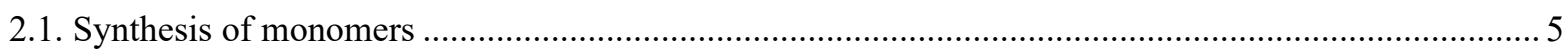

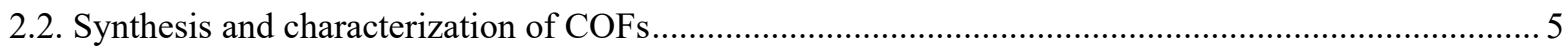

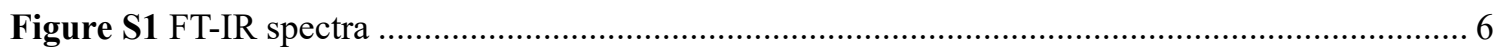

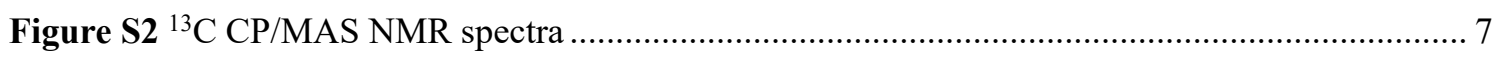

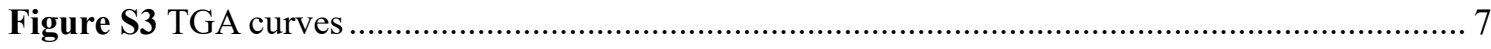

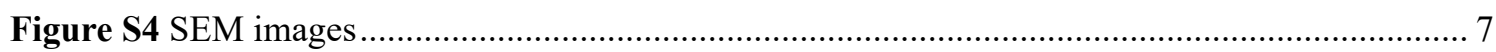

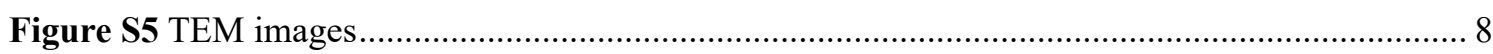

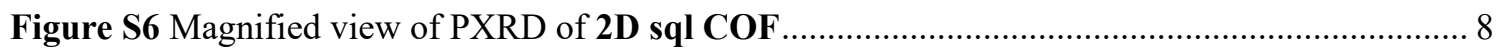

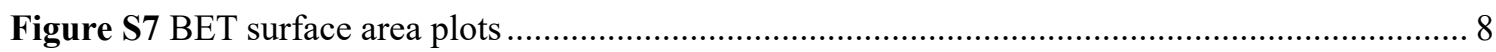

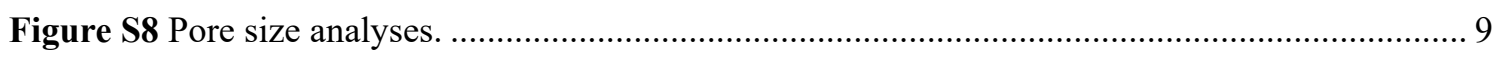

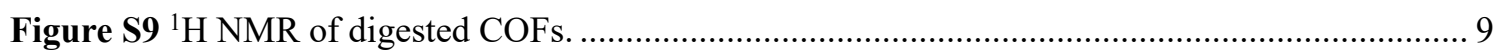

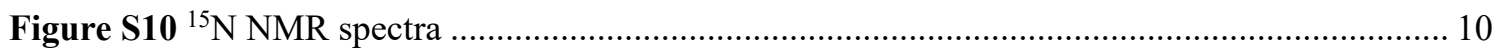

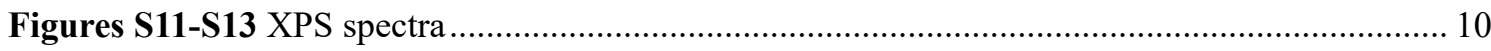

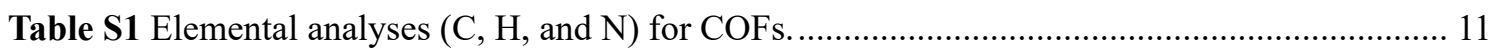

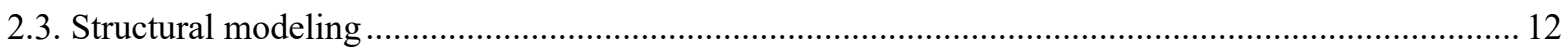

2.3.1. Figures S14-S18 Fully connected topologies for COFs ....................................................12-14

2.3.2. Figures S19-S48 Partially connected topologies for COFs ....................................................... 14-24

Table S2 Pawley and Rietveld refinement results for COFs........................................................ 25

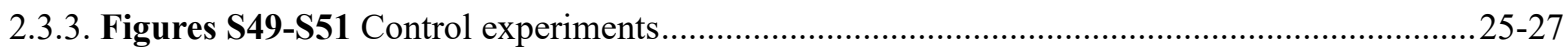

Figure S52 Coverage-dependent adsorption enthalpies and selectivities of equimolar binary

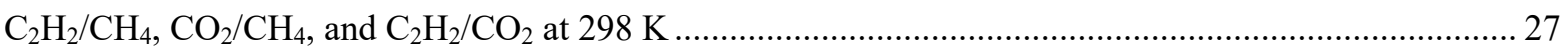

Figures S53-S56 GCMC simulation results. ....................................................................2 28-29

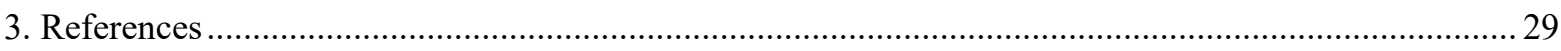




\section{Materials and methods}

All chemicals are commercially available, and used without further purification. All solvents were dried and distilled according to conventional methods.

Powder X-ray diffraction (PXRD): PXRD patterns were collected on an X-ray diffraction (XRD) system (D/max-Ultima IV, Japan) using $\mathrm{Cu} \mathrm{K \alpha}$ radiation.

Fourier transform infrared (FT-IR): IR spectrum was measured on an IR spectrometer (Nicolet 6700) between the ranges of 4000 to $400 \mathrm{~cm}^{-1}$.

Solid-state nuclear magnetic resonance (ssNMR): Solid-state nuclear magnetic resonance (NMR) data were performed on a Bruker AVANCE III 600 spectrometer with crosspolarization magic-angle-spinning (CP/MAS) at a resonance frequency of $150.9 \mathrm{MHz} \cdot{ }^{13} \mathrm{C}$ CP/MAS NMR spectra were recorded using a $4 \mathrm{~mm}$ MAS probe and a spinning rate of $12 \mathrm{kHz}$. A contact time of $4 \mathrm{~ms}$ and a recycle delay of $2 \mathrm{~s}$ were used for the ${ }^{13} \mathrm{C}$ CP/MAS NMR measurement. The chemical shifts of ${ }^{13} \mathrm{C}$ were externally referenced to tetramethylsilane (TMS). ${ }^{15} \mathrm{~N} \mathrm{CP} / \mathrm{MAS}$ NMR spectra were recorded using a $4 \mathrm{~mm}$ MAS probe and a spinning rate of $10 \mathrm{kHz}$.

Elemental analyses: Elemental analyses were performed on an Elementar vario MICRO UNICUBE series CHN elemental analyzer.

Solution nuclear magnetic resonance (NMR): Liquid state ${ }^{1} \mathrm{H}$ nuclear magnetic resonance spectroscopy was collected on a Varian Mercury Plus 400 NMR Spectrometer.

Scanning electron microscope (SEM): SEM images were collected using a Nova NanoSEM 450 system.

Transmission electron microscope (TEM): TEM images were obtained with a Talos SFEG.

Thermogravimetric analysis (TGA): TGA was performed using a TA Q800 under flowing $\mathrm{N}_{2}$ with $10 \mathrm{~K} \mathrm{~min}^{-1}$ ramp rate. Samples were heated in a Platinum pan $\left(800{ }^{\circ} \mathrm{C}, 10{ }^{\circ} \mathrm{C} \mathrm{min}{ }^{-1}\right)$ under a $\mathrm{N}_{2}$ flux $\left(60 \mathrm{~mL} \mathrm{~min}^{-1}\right)$.

Gas sorption: The samples were activated under vacuum for 12 hours at $120^{\circ} \mathrm{C}$. Gas adsorption experiments at $298 \mathrm{~K}$ were performed by using JW-BK200 surface area analyzer. A circulation constant temperature water bath was used to stabilize the temperature at $298 \mathrm{~K}$ during the test.

X-ray photoelectron spectroscopy (XPS): X-ray photoelectron spectroscopy data were collected using a Thermo ESCALAB 250Xi.

\section{Crystal structure modeling:}

Structural modeling of COFs was generated using the Materials Studio ${ }^{\mathrm{S} 1}$ program employing the Building (Crystal) module, possible topologies for all combination for $\mathrm{C}_{6}+\mathrm{T}_{\mathrm{d}} / \mathrm{C}_{4}$, or the subsets of $\mathrm{C}_{6}+\mathrm{T}_{\mathrm{d}} / \mathrm{C}_{4}$, such as $\mathrm{C}_{4} / \mathrm{C}_{3} / \mathrm{C}_{2}+\mathrm{T}_{\mathrm{d}} / \mathrm{C}_{4}$ were built according to RCSR database $^{\mathrm{S} 2}$. The lattice model was geometrically optimized using force-filed based method (Forcite molecular dynamics module) and SCC-DFTB (DFTB + module). The Pawley fitting (Reflex module) was performed to optimize the lattice parameters iteratively until the Rwp value converges and the overlay of the observed with refined profiles shows good agreement. Powder indexing and Rietveld refinement were performed using EXPO2014 ${ }^{\mathrm{S} 3}$, various topology structures were illustrated by VESTA software ${ }^{\mathrm{s} 4}$. Theorical surface area for COFs 
were analyzed and calculated by Zeo++ ${ }^{\mathrm{S5}}$ using $\mathrm{N}_{2}$ as probe molecule.

\section{Isosteric heat of adsorption:}

A Virial equation comprising of the temperature-independent parameters $a_{i}$ and $b_{i}$ was employed to calculate the enthalpies of adsorption for $\mathrm{C}_{2} \mathrm{H}_{2}, \mathrm{CH}_{4}$ and $\mathrm{CO}_{2}$, which measured at two different temperatures $273 \mathrm{~K}$ and $298 \mathrm{~K}$.

$\ln P=\ln N+\frac{1}{T} \sum_{i}^{m} u_{i} N_{i}+\sum_{j}^{n} b_{j} N_{j}$

$Q_{s t}=-R \sum_{i=0}^{m} a_{i} N_{i}$

$P$ is the pressure (mmHg), $N$ is the adsorbed quantity $\left(\mathrm{mmol} \mathrm{g}^{-1}\right), T$ is the temperature $(\mathrm{K}), R$ is the gas constant $\left(8.314 \mathrm{~J} \mathrm{~K}^{-1} \mathrm{~mol}^{-1}\right), a i$ and $b i$ are virial coefficients, and $\mathrm{m}$ and $\mathrm{n}$ represent the number of coefficients required to adequately describe the isotherms (herein, $m=5$, and $n$ $=2)$.

\section{Calculation of selectivity via ideal adsorption solution theory (IAST):}

The gas adsorption isotherms were first fitted to a dual-site Langmuir-Freundlich (DSLF) model (eqn (3)), where is the amount of adsorbed gas $q\left(\mathrm{mmol} \mathrm{g}^{-1}\right), P$ is the bulk gas phase pressure (atm), qsat is the saturation amount $\left(\mathrm{mmol} \mathrm{g}^{-1}\right), b$ is the Langmuir-Freundlich parameter $\left(\mathrm{atm}^{-\alpha}\right)$, and $\alpha$ is the Langmuir-Freundlich exponent (dimensionless) for two adsorption sites $\mathrm{A}$ and $\mathrm{B}$ indicating the presence of weak and strong adsorption sites.

$\mathrm{q}=q_{A, s a t} \frac{b_{A} p}{1+b_{A} p}+q_{B, s a t} \frac{b_{B} p}{1+b_{B} p}$

IAST starts from the Raoult's Law type of relationship between the fluid and adsorbed phase (4) and (5), where $P_{i}$ is the partial pressure of component $i$ (atm), $P$ is the total pressure (atm), and $y_{i}$ and $x_{i}$ represent mole fractions of component $i$ in gas and the adsorbed phase (dimensionless). $P_{i}^{0}$ is the equilibrium vapour pressure (atm).

$\mathrm{P}_{i}=P y_{i}=P_{i}^{0} x_{i}$

$\sum_{i=1}^{n} x_{i}=\sum_{i=1}^{n} \frac{P_{i}}{P_{i}^{0}}=1$

In IAST, $P$ is defined by relating to spreading pressure $\pi(6)$, where $\pi$ is the spreading pressure, $\mathrm{S}$ is the specific surface area of the adsorbent $\left(\mathrm{m}^{2} \mathrm{~g}^{-1}\right), \mathrm{R}$ is the gas constant $\left(8.314 \mathrm{~J} \mathrm{~K}^{-1} \mathrm{~mol}^{-}\right.$ $\left.{ }^{1}\right)$, $\mathrm{T}$ is the temperature $(\mathrm{K})$, and $q_{i}\left(P_{i}\right)$ is the single component equilibrium obtained from isotherms $\left(\mathrm{mmol} \mathrm{g}^{-1}\right)$.

$\frac{\pi S}{R T}=\int_{0}^{P^{0}} i \frac{q_{i}\left(P_{i}\right)}{P_{i}} d P_{t}=\Pi($ condtant $)$

For a DSLF model, we have an analytical expression for the integral (7). The isotherm parameters are derived from the previous fitting. For a binary component system, the unknowns will be $\Pi, P$, and which can be obtained by simultaneously solving eqn (5) and (7).

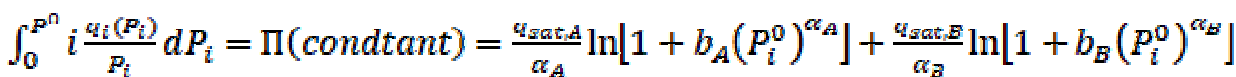

The adsorbed amount of each compound in a mixture is (8) and (9), where $q$ is the adsorbed amount of component $\left(\mathrm{mmol} \mathrm{g}^{-1}\right)$, and is the total adsorbed amount $\left(\mathrm{mmol} \mathrm{g}^{-1}\right)$.

$q_{i}^{\text {mix }}=x_{i} q_{t}$ 
$\frac{1}{q T}=\sum_{i=1}^{n} \frac{x_{i}}{q_{i}\left(P_{i}^{0}\right)}$

The adsorption selectivities $S_{a d s}$ were calculated using eqn (10). In this study, IAST calculations were carried out assuming a binary mixed gas with a molar ratio of 50:50 at 273 $\mathrm{K}$ and $298 \mathrm{~K}$ and pressures up to $1 \mathrm{~atm}$.

$S_{a d s}=\frac{q_{1} / q_{2}}{p_{1} / p_{2}}$

\section{Computational simulation studies of gases adsorption:}

Grand Canonical Monte Carlo (GCMC) simulations were carried out using the Sorption module of Materials Studio 8.0 package. The Locate and Metropolis methods were used to predict the possible binding sites of $\mathrm{C}_{2} \mathrm{H}_{2}, \mathrm{CO}_{2}$, and $\mathrm{CH}_{4}$ onto the framework. The unit cell framework of COFs was constructed from simulated X-ray diffraction data. During the simulation, all gas molecules and the frameworks were considered as rigid, and periodic boundary conditions were applied in all three directions. All parameters including the partial charges were assigned by the COMPASS force field embedded in the Sorption module.

\section{Synthesis and general procedures}

\subsection{Synthesis of monomers}

Hexaldehydephenyl benzene $(\mathbf{H F B})^{\mathbf{S 6}}$ and amine monomers Tetrakis(4aminophenyl)ethane (TPE-NH2), ${ }^{\mathbf{S 6}} \quad$ 3,3',5,5'-tetra(p-aminophenyl)-bimesitylene (BMTA) $)^{\mathbf{S 7}}$ were synthesized according to the published methods.

\subsection{Synthesis and characterization of COFs}<smiles>O=Cc1ccc(-c2c(-c3ccc(C=O)cc3)c(-c3ccc(C=O)cc3)c(-c3ccc(C=O)cc3)c(-c3ccc(C=O)cc3)c2-c2ccc(C=O)cc2)cc1</smiles>

HFB

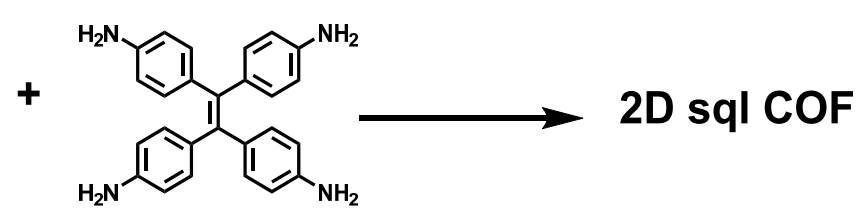

TPE- $\mathrm{NH}_{2}$

2D sql COF: HFB $(21.1 \mathrm{mg}, 0.03 \mathrm{mmol})$ and TPE-NH2 $(11.8 \mathrm{mg}, 0.03 \mathrm{mmol})$ were weighed into a glass ampoule with $o$-dichlorobenzene $(0.8 \mathrm{~mL})$ and $n$-butyl alcohol $(0.8 \mathrm{~mL})$. The solution was ultrasound for 5 minutes to obtain yellow turbid solution. $6 \mathrm{M}$ acetic acid $(0.3 \mathrm{~mL})$ was pure into the glass ampoule as catalyst. The glass ampoule was flash frozen at $77 \mathrm{~K}$ using the liquid nitrogen bath and degassed by freeze-pump-thaw three times, and then sealed. The glass ampoule was placed at $120^{\circ} \mathrm{C}$ for 3 days. The yellow solid was isolated by 
centrifugation and washed with acetone $(3 \times 10 \mathrm{~mL})$ and then filtered. The sample was then transferred to vacuum chamber and evacuated to 20 mTorr at $80^{\circ} \mathrm{C}$ for $24 \mathrm{~h}$, yielding yellow powder 2D sql COF (Yield: $26.1 \mathrm{mg}, 85 \%$ ).<smiles>O=Cc1ccc(-c2c(-c3ccc(C=O)cc3)c(-c3ccc(C=O)cc3)c(-c3ccc(C=O)cc3)c(-c3ccc(C=O)cc3)c2-c2ccc(C=O)cc2)cc1</smiles>

HFB

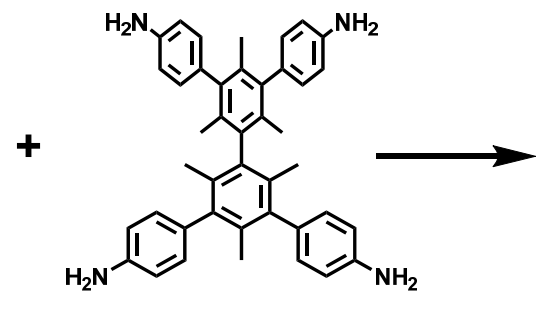

BMTA

3D pts COF: HFB (21.1 mg, $0.03 \mathrm{mmol})$ and BMTA (18.1 mg, $0.03 \mathrm{mmol})$ were weighed into a glass ampoule with $o$-dichlorobenzene $(0.8 \mathrm{~mL})$ and $n$-butyl alcohol $(0.8 \mathrm{~mL})$. The solution was ultrasound for 5 minutes to obtain yellow turbid solution. $6 \mathrm{M}$ acetic acid (0.3 $\mathrm{mL}$ ) was pure into the glass ampoule as catalyst. The glass ampoule was flash frozen at $77 \mathrm{~K}$ using the liquid nitrogen bath and degassed by freeze-pump-thaw three times, and then sealed. The glass ampoule was placed at $120{ }^{\circ} \mathrm{C}$ for 3 days. The yellow solid was isolated by centrifugation and washed with acetone $(3 \times 10 \mathrm{~mL})$ and then filtered. The sample was then transferred to vacuum chamber and evacuated to $20 \mathrm{mTorr}$ at $80^{\circ} \mathrm{C}$ for $24 \mathrm{~h}$, yielding yellow powder 3D pts COF (Yield: $30.3 \mathrm{mg}, 82 \%$ ).

(a)

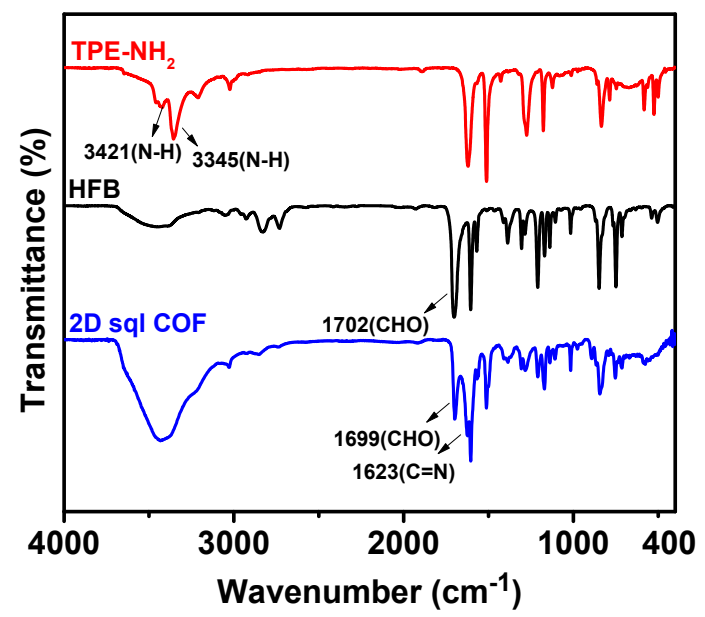

(b)

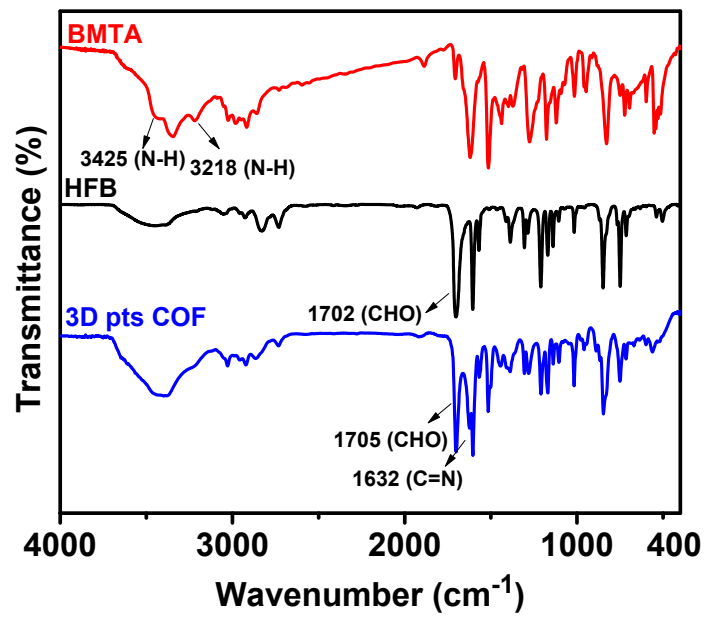

Figure S1. FT-IR spectra of (a) 2D sql COF and (b) 3D pts COF 
(a)

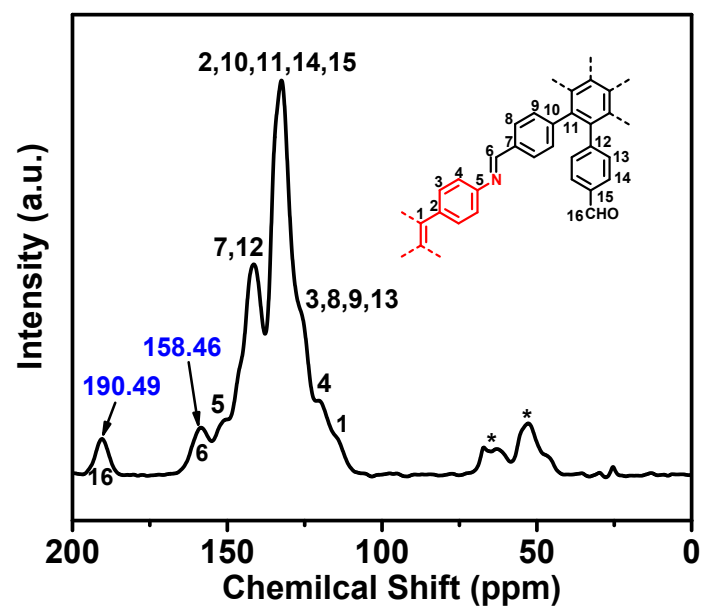

(b)

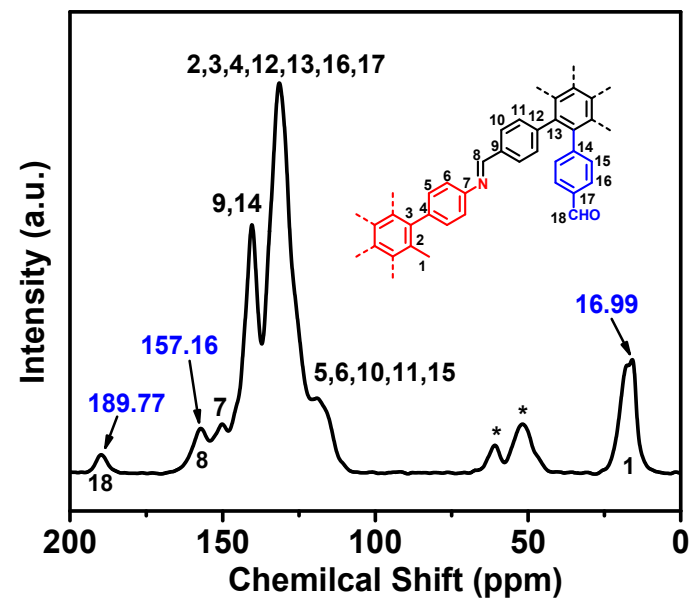

Figure S2. ${ }^{13} \mathrm{C} \mathrm{CP} /$ MAS NMR spectra for (a) 2D sql COF and (b) 3D pts COF

(a)

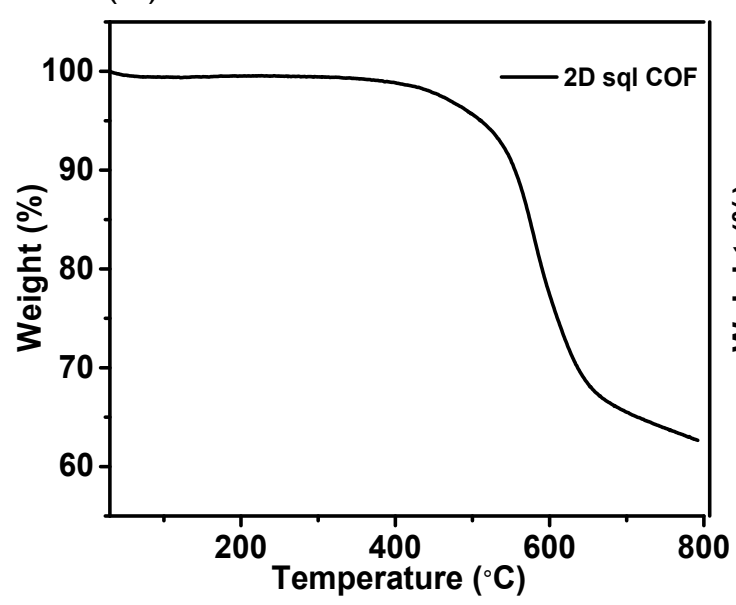

(b)

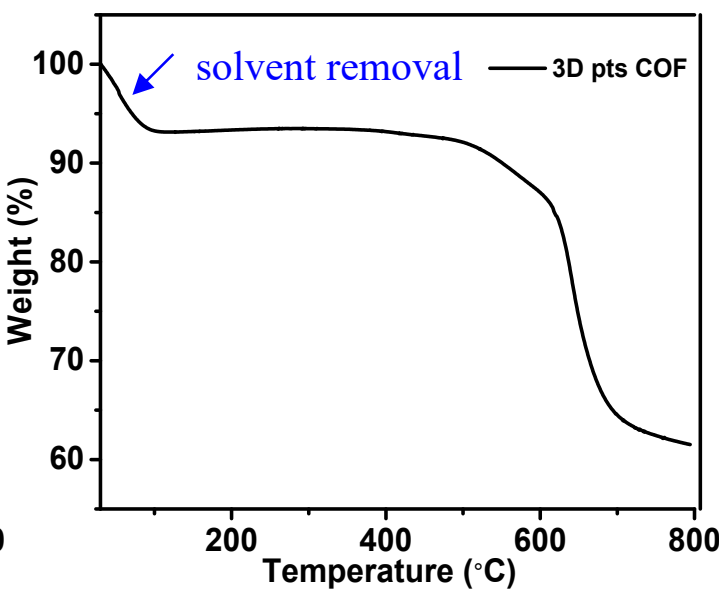

Figure S3. TGA curves of (a) 2D sql COF and (b) 3D pts COF

(a)

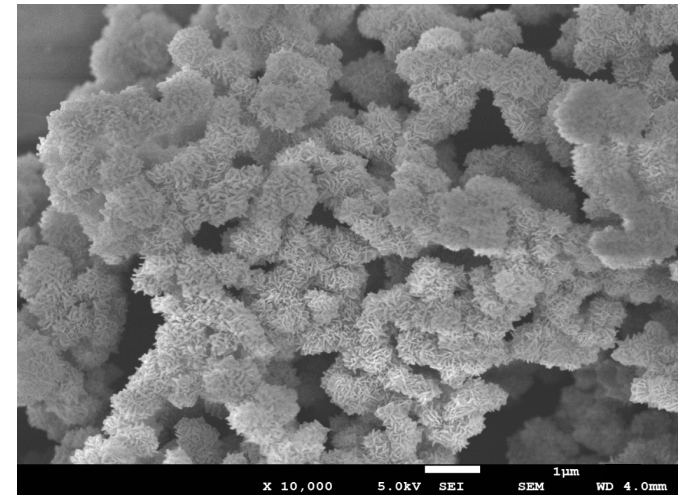

(b)

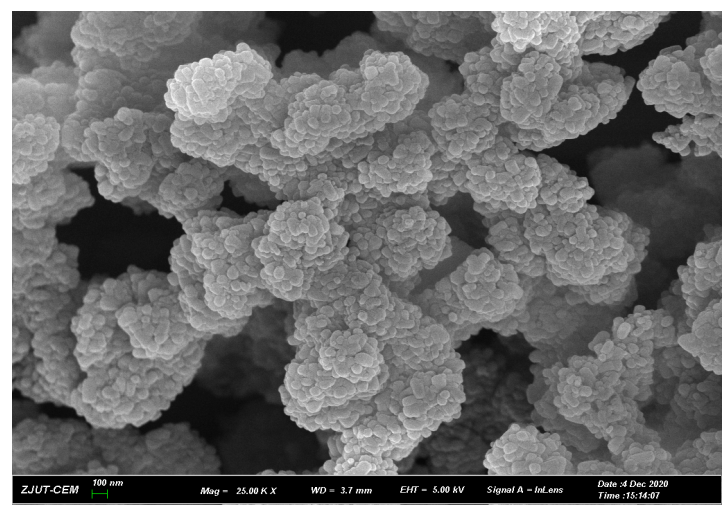

Figure S4. SEM images of (a) 2D sql COF and (b) 3D pts COF 
(a)

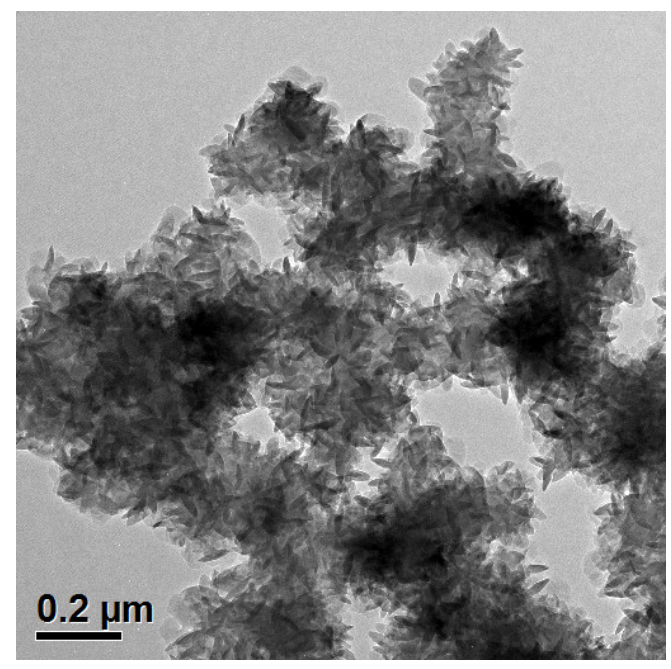

(b)

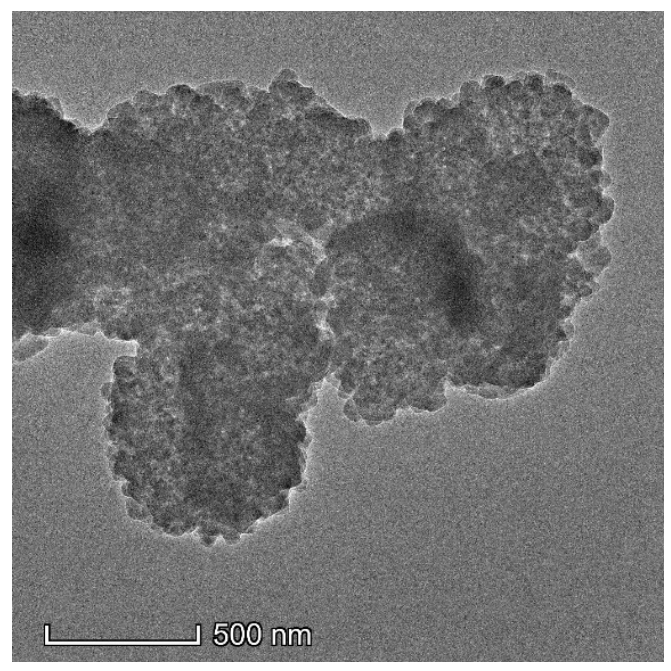

Figure S5. TEM images of (a) 2D sql COF and (b) 3D pts COF

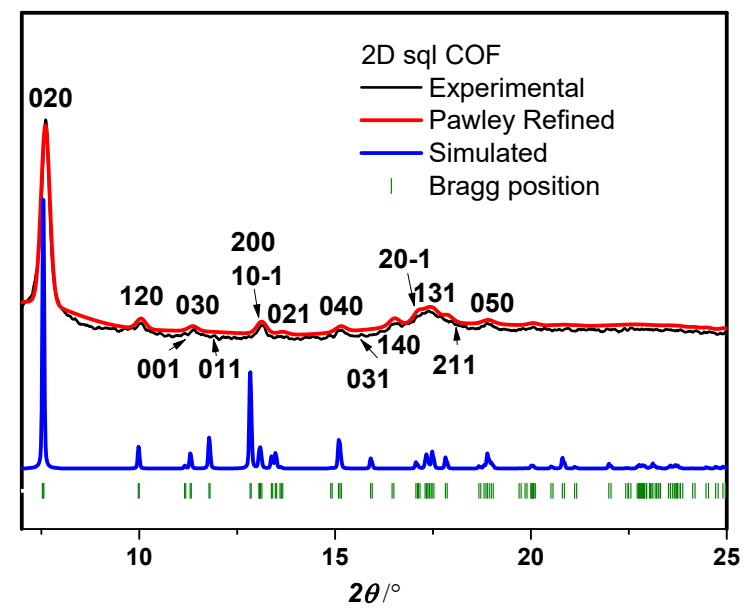

Figure S6. Magnified view of PXRD of 2D sql COF, properly demonstrating the spectral Bragg positions.
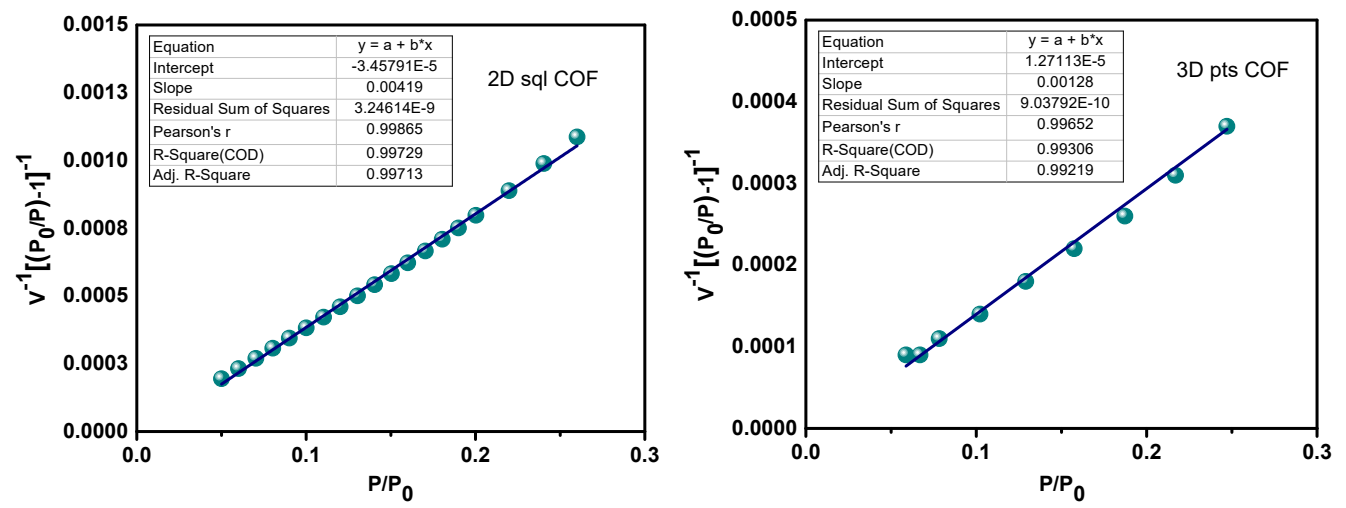

Figure S7. BET surface area plots for 2D sql COF and 3D pts COF. 
(a)

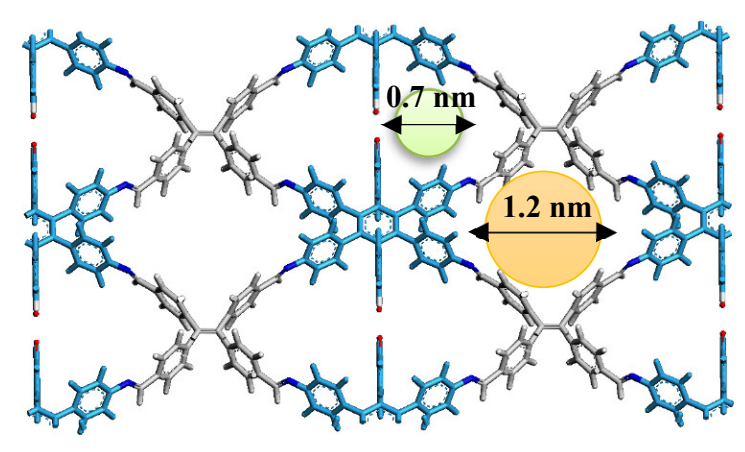

(b)

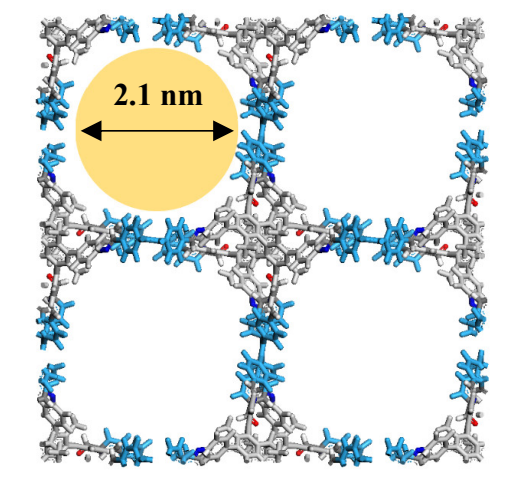

Figure S8. Pore size analyses from simulated structures for (a) 2D sql COF and (b) 3D pts COF.

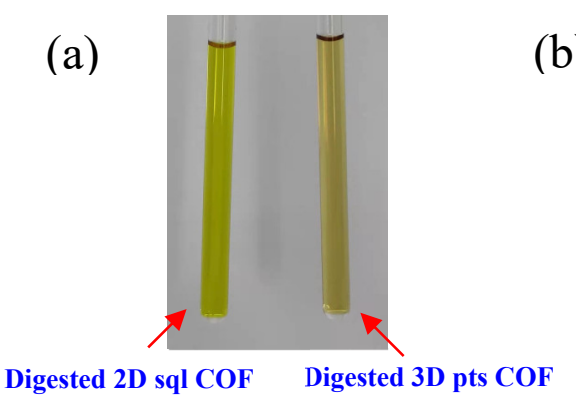

(b)
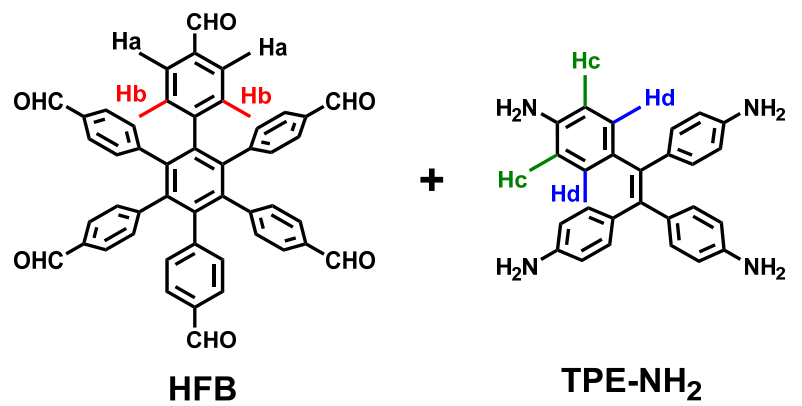

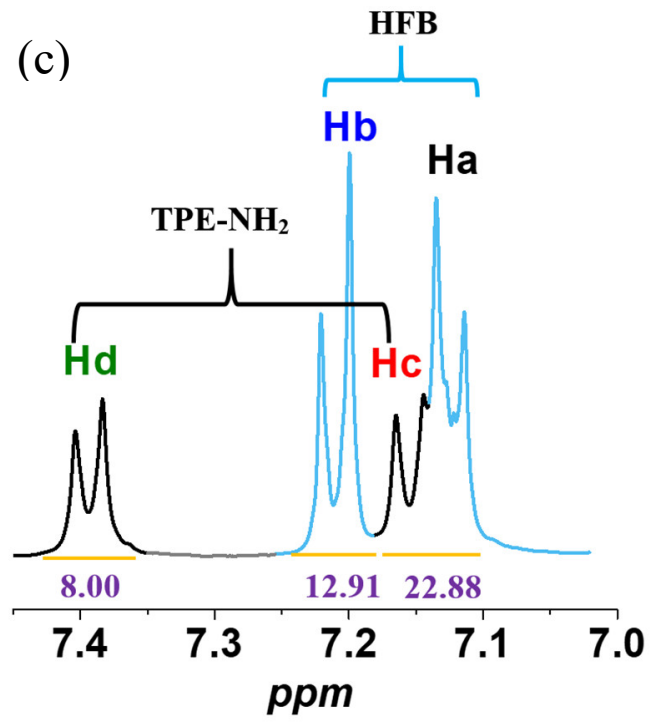

Figure S9. Pictures of digested (a) 2D sql COF and 3D pts COF in NMR tubes. (b) Protons labeled in monomers from digested COFs (c) 1H NMR of digested 2D sql COF.

"Note: The activated 2D sql COF or 3D pts COF $(15 \mathrm{mg})$, DMSO- $d^{6}(0.5 \mathrm{~mL})$ and DCl $(0.1 \mathrm{~mL})$ were added into a $10 \mathrm{~mL}$ Pyrex tube. The mixture was heated at $70{ }^{\circ} \mathrm{C}$ for $4 \mathrm{~h}$ under 
a nitrogen atmosphere. After cooling to room temperature, the obtained clear solution (Figure S9a) was subjected to ${ }^{1} \mathrm{HNMR}$ spectroscopy analysis. Both COFs can be fully digested in DCl/ DMSO- $d^{6}$, which means that the linker ratios calculated from the solution is equal to that of COF structure itself. The mole ratio of digested HFB to TPE-NH $\mathbf{N H}_{2}$ can be calculated as the formula:

$$
\mathrm{n}(\mathrm{HFB}) / \mathrm{n}\left(\mathrm{TPE}-\mathrm{NH}_{2}\right)=\frac{\left(\int H a\right) / 12}{\left(\int H c\right) / 8}=\frac{2 \int H a}{3 \int H c} \text {, apparently } \mathrm{Ha}=\mathrm{Hb}, \mathrm{Hc}=\mathrm{Hd} \text {, }
$$

As shown in above Figure S9c of integration, two distinct sets of profiles attributed to Ha $(\mathrm{Hb})$ and $\mathrm{Hc}(\mathrm{Hd})$ are revealed, and overlapping region are the combination of $\mathrm{Ha}$ and $\mathrm{Hc}$, which can be simply confirmed by either symmetric profiles of two sets of protons, or the integration results signifying that $\mathrm{Ha}+\mathrm{Hc}$ almost equals with that of $\mathrm{Hb}+\mathrm{Hd}$.

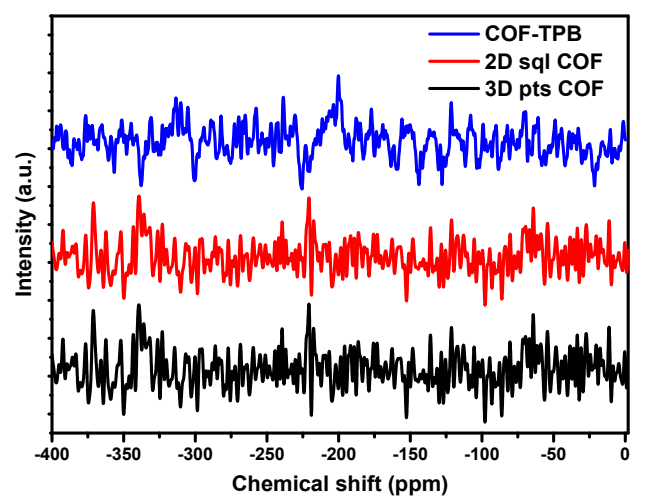

Figure S10. ${ }^{15} \mathrm{~N}$ NMR spectra for COFs.

"Note: Due to the extremely low N content in these COFs, we could not obtain N signal from their ${ }^{15} \mathrm{~N}$ NMR spectra, despite the fact that we have collected the ${ }^{15} \mathrm{~N}$ NMR spectrum for 72 hours for every COF.

(a)

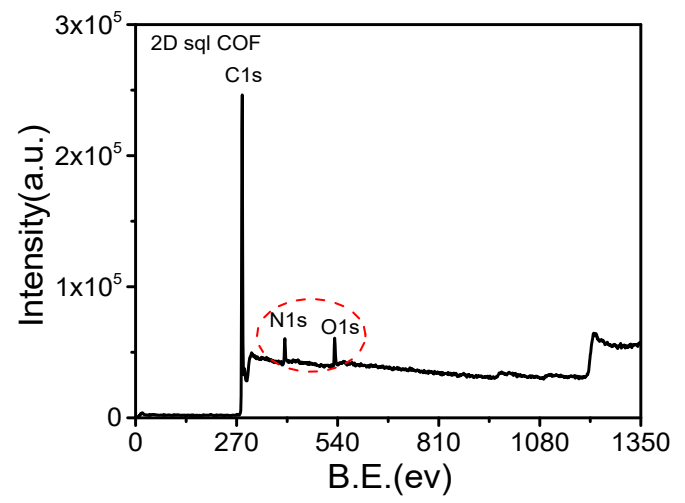

(b)

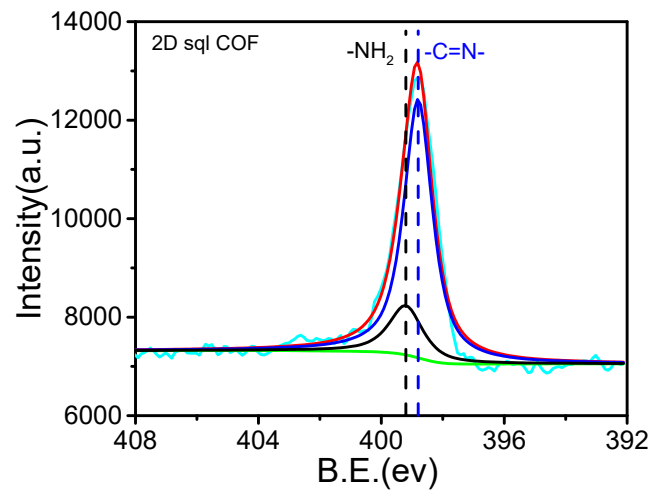

Figure S11. XPS spectra for 2D sql COF. 
(a)

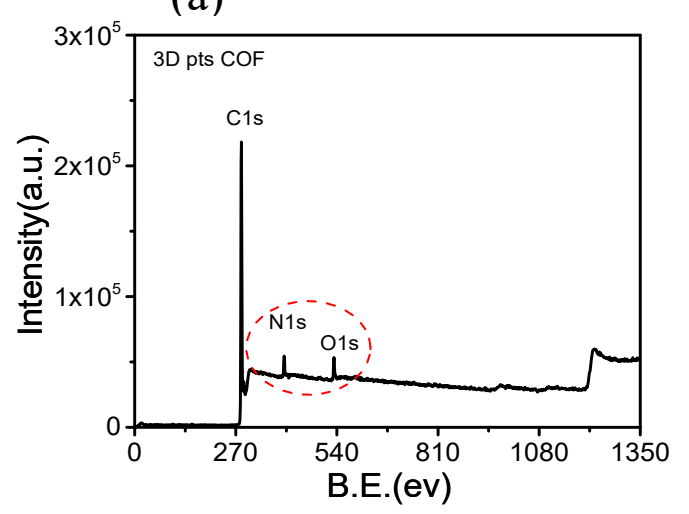

(b)

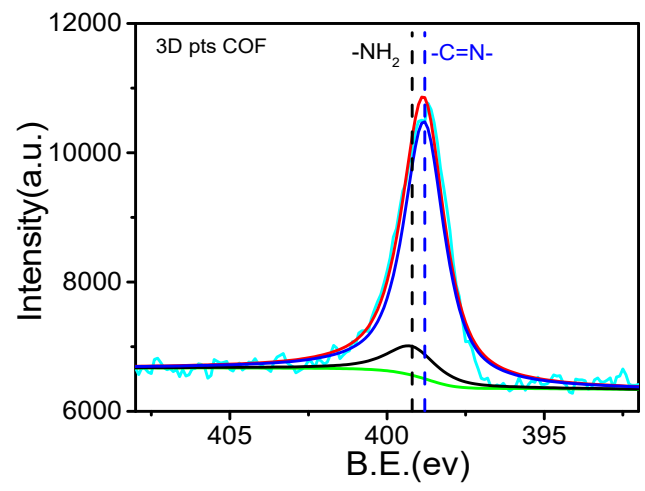

Figure S12. XPS spectra for 3D pts COF.

(a)

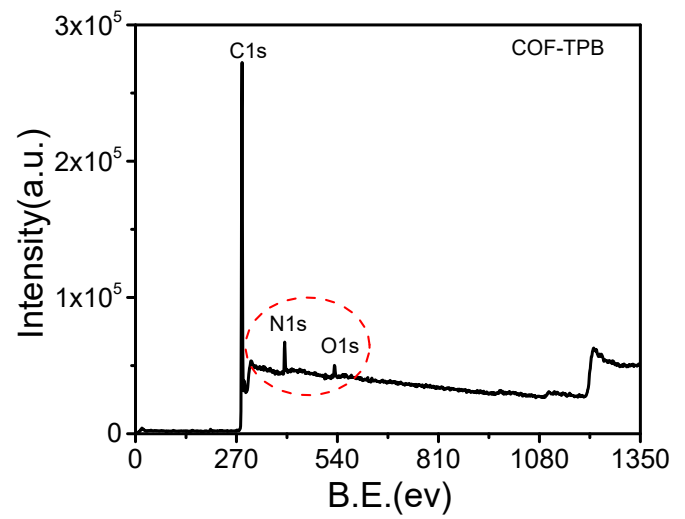

(b)

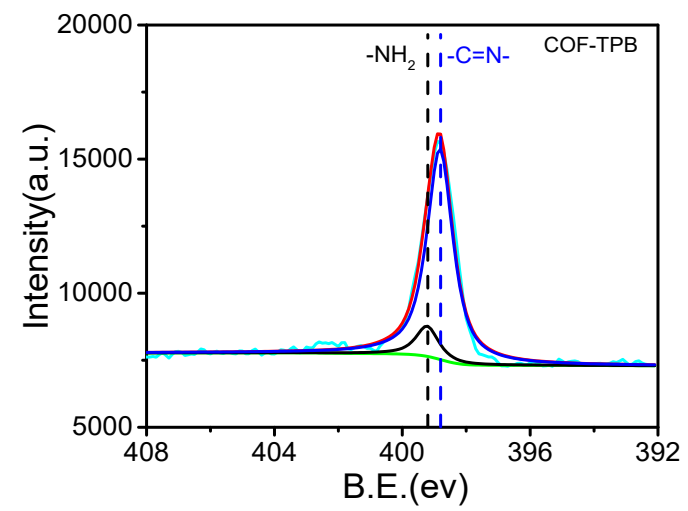

Figure S13. XPS spectra for COF-TPB.

"Note: The presence of residual amine groups in Figures S11b/S12b/S13b are attributed to the unreacted amine residing onto the surface of COF particles. The relatively lower amine and oxygen contents are revealed for all three COFs. It should be noted that the marginal amine/aldehydes onto the COF particles in this respect are not involved in the construction for $\mathrm{COF}$, the ratio of two monomer has been confirmed as nearly 1:1 from digested ${ }^{1} \mathrm{H}$ NMR results, and we also notice that the nearly but not exactly 1:1 ratio may be just due to the unreacted amine and aldehydes attaching onto the surface of COF particles, which is clearly demonstrated and calculated in Figure S9.

Table S1. Elemental analyses (C, H, and N) for COFs.

\begin{tabular}{|c|c|c|c|}
\hline \multirow{2}{*}{ Sample } & \multicolumn{3}{|c|}{ \% Caculated/Found } \\
\cline { 2 - 4 } & $\mathrm{C}$ & $\mathrm{H}$ & $\mathrm{N}$ \\
\hline 2D sql COF & $86.87 / 82.07$ & $4.53 / 4.76$ & $5.48 / 5.32$ \\
\hline 3D pts COF & $87.63 / 84.44$ & $5.23 / 5.35$ & $4.54 / 4.78$ \\
\hline COF-TPB & $88.43 / 81.63$ & $4.70 / 5.16$ & $6.87 / 5.77$ \\
\hline
\end{tabular}




\subsection{Structural modeling}

\subsubsection{Fully connected topologies for COFs}

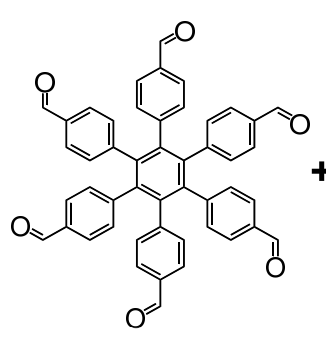

HFB

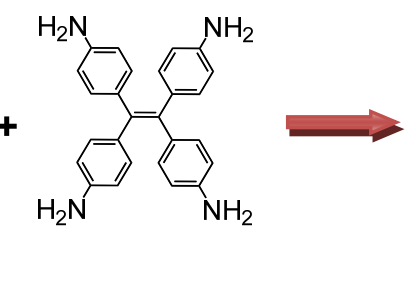

TPE-NH

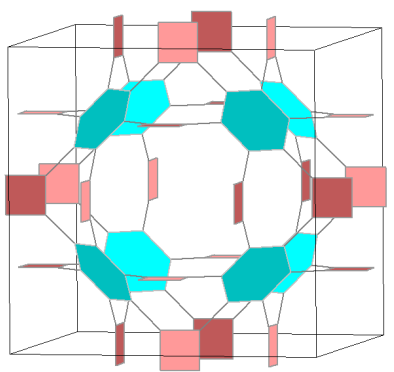

she-a

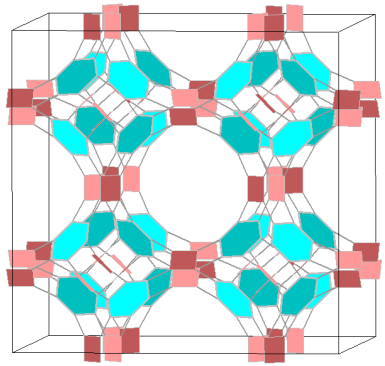

nts-a
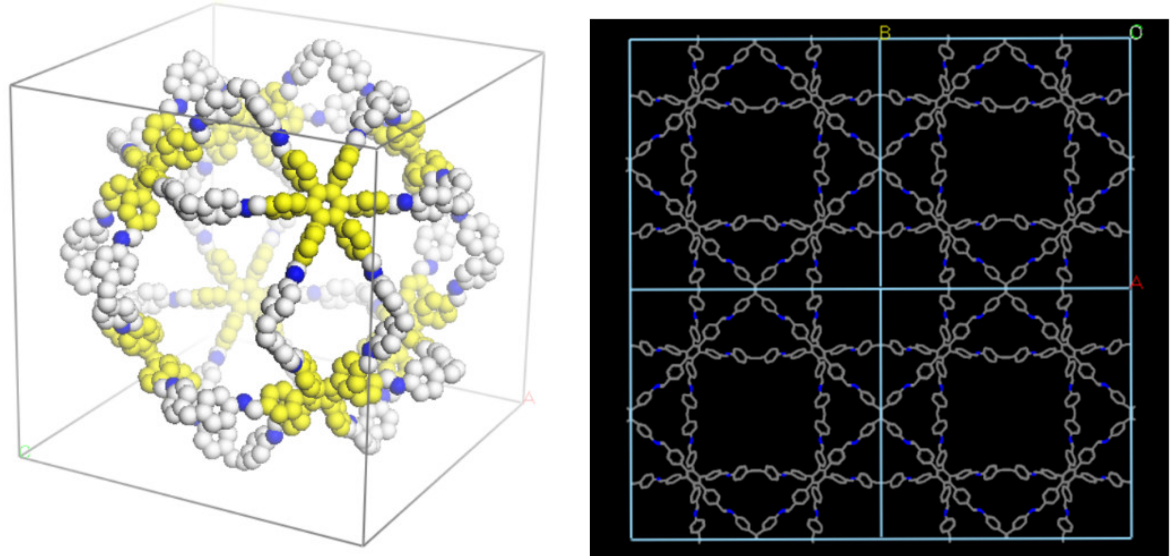

Figure S14. Structural illustration for possible 3D she-a topology built from HFB and TPE$\mathrm{NH}_{2}$.
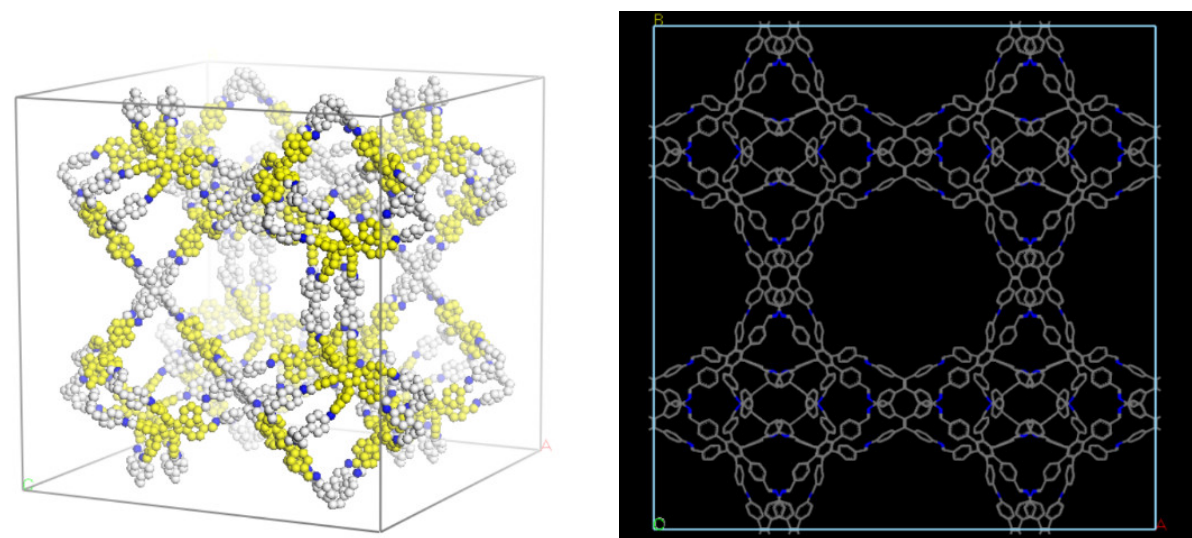

Figure S15. Structural illustration for possible 3D nts-a topology built from HFB and TPE$\mathrm{NH}_{2}$. 


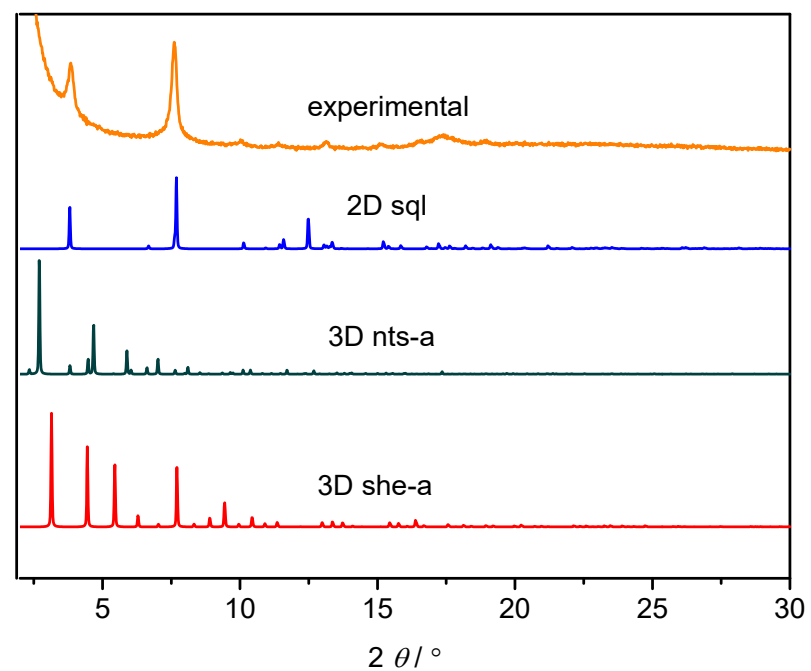

Figure S16. PXRD patterns $(\mathrm{Cu} \mathrm{K} \alpha)$ for possible topologies of $2 \mathrm{D}$ and 3D structures constructed from HFB and TPE-NH2.<smiles>O=Cc1ccc(-c2c(-c3ccccc3)c(-c3ccc(C=O)cc3)c(-c3ccc(C=O)cc3)c(-c3ccc(C=O)cc3)c2-c2ccc(C=O)cc2)cc1</smiles>

HFB<smiles>Cc1c(-c2ccc(N)cc2)c(C)c(-c2ccc(N)cc2-c2ccc(N)cc2)c(C)c1-c1ccc(N)cc1</smiles>

BMTA

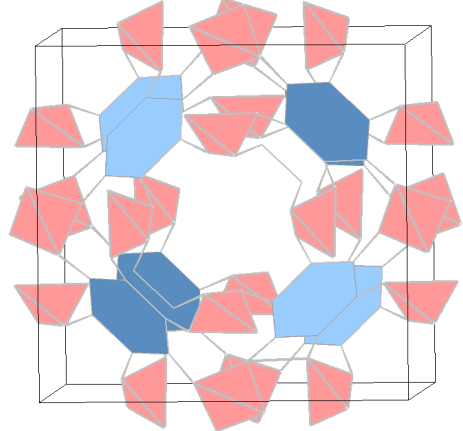

(T)
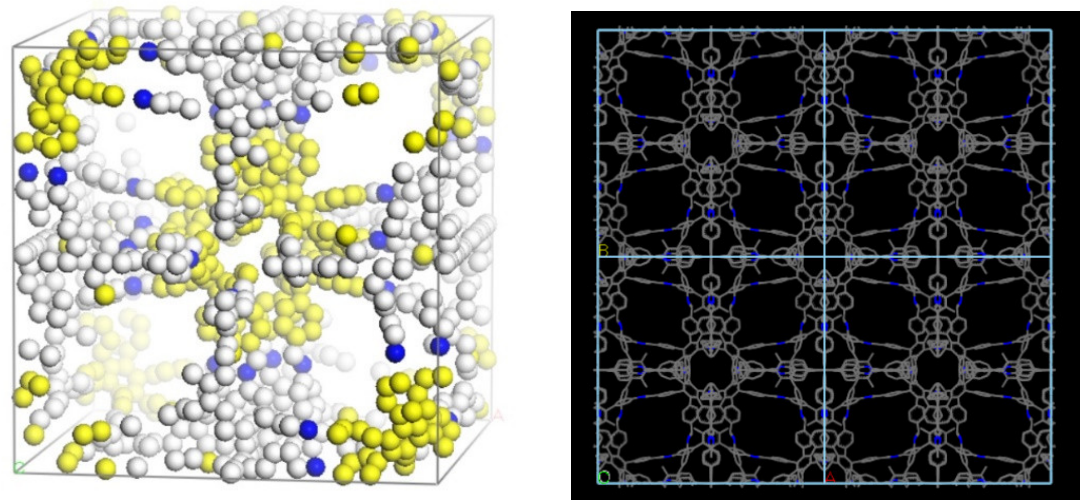

Figure S17. Structural illustration for possible 3D het-a topology built from HFB and BMTA. 


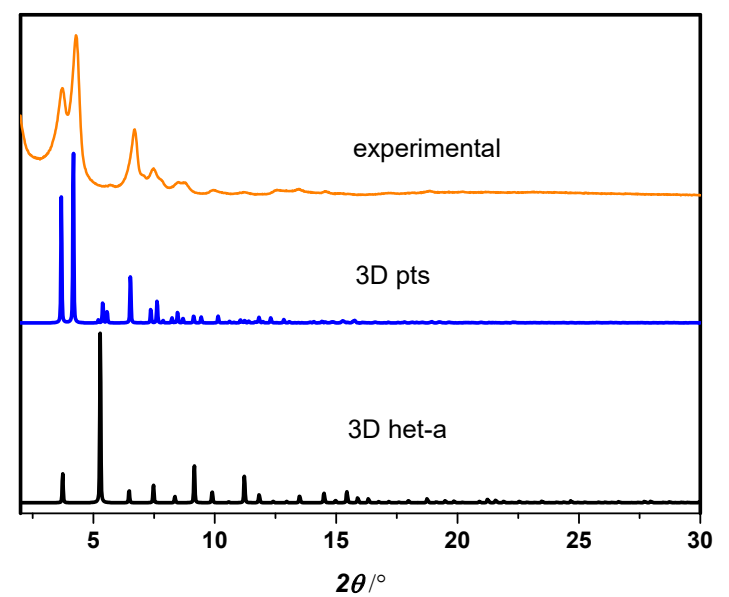

Figure S18. PXRD patterns $(\mathrm{Cu} \mathrm{K} \alpha)$ for possible topologies of 3D structures constructed from HFB and BMTA.

\subsubsection{Partially connected topologies for COFs}

1) $\mathrm{C}_{4} /$ tetratopic $+\mathrm{C}_{4} /$ tetratopic:

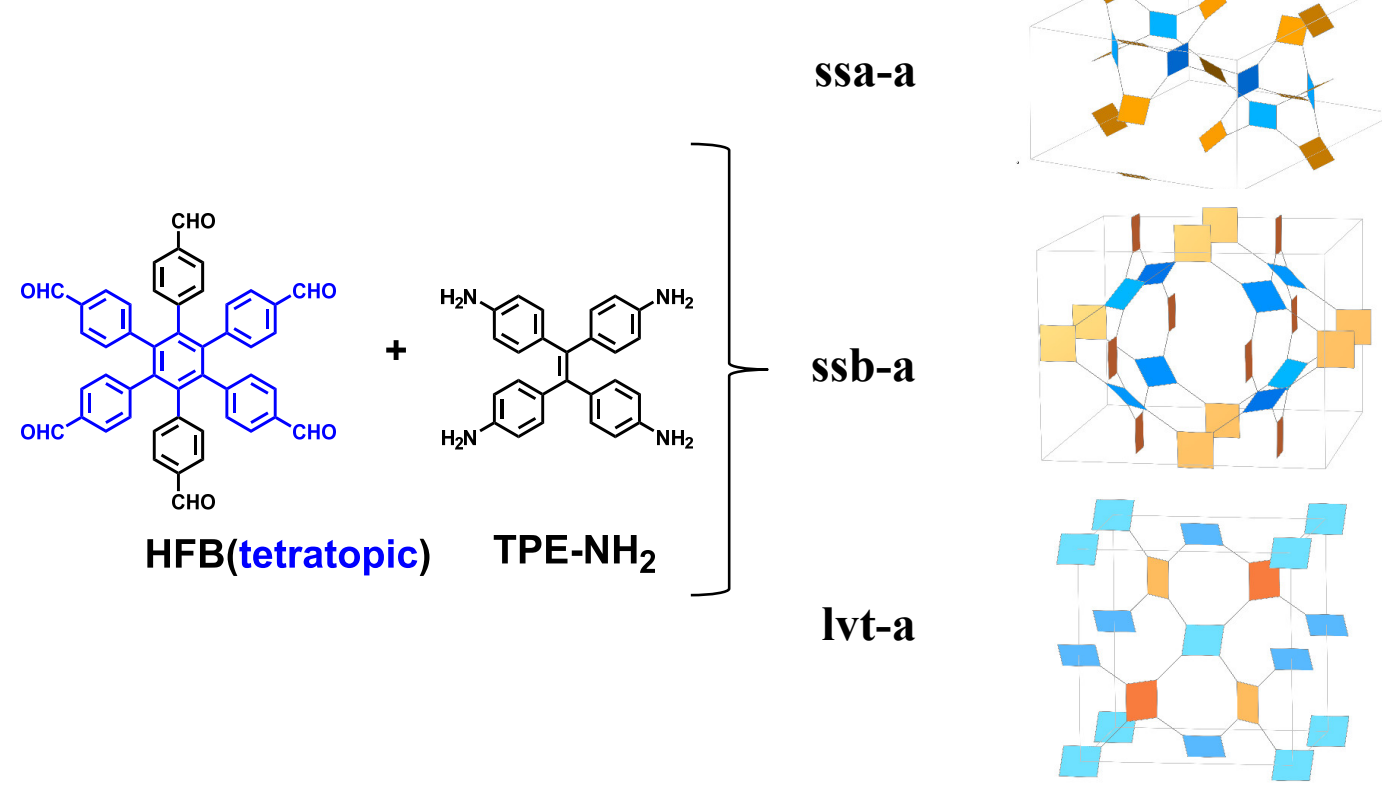

Figure S19. Possible partially connected topologies of 3D structures constructed from HFB (tetratopic) and TPE-NH2. 

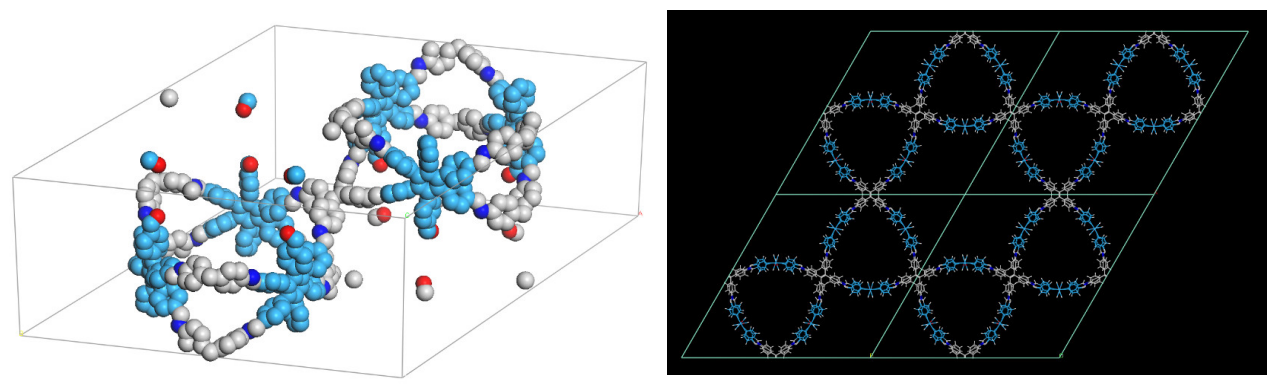

Figure S20. Structural illustration for possible 3D ssa-a topology built from HFB (tetratopic) and TPE-NH2.
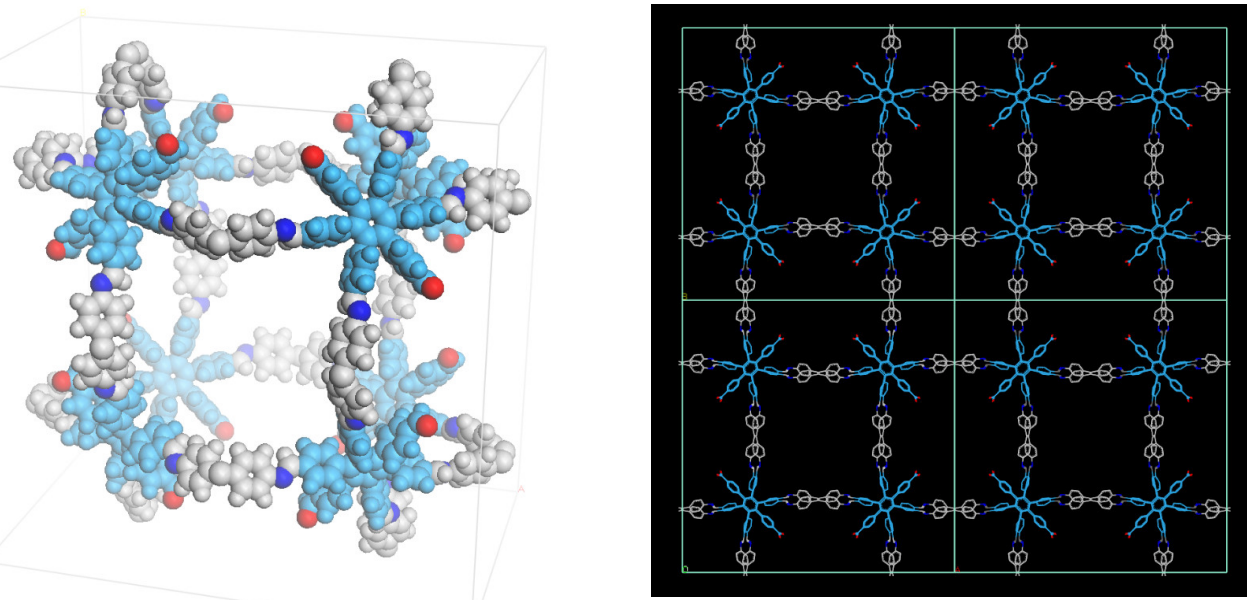

Figure S21. Structural illustration for possible 3D ssb-a topology built from HFB (tetratopic) and TPE-NH2.
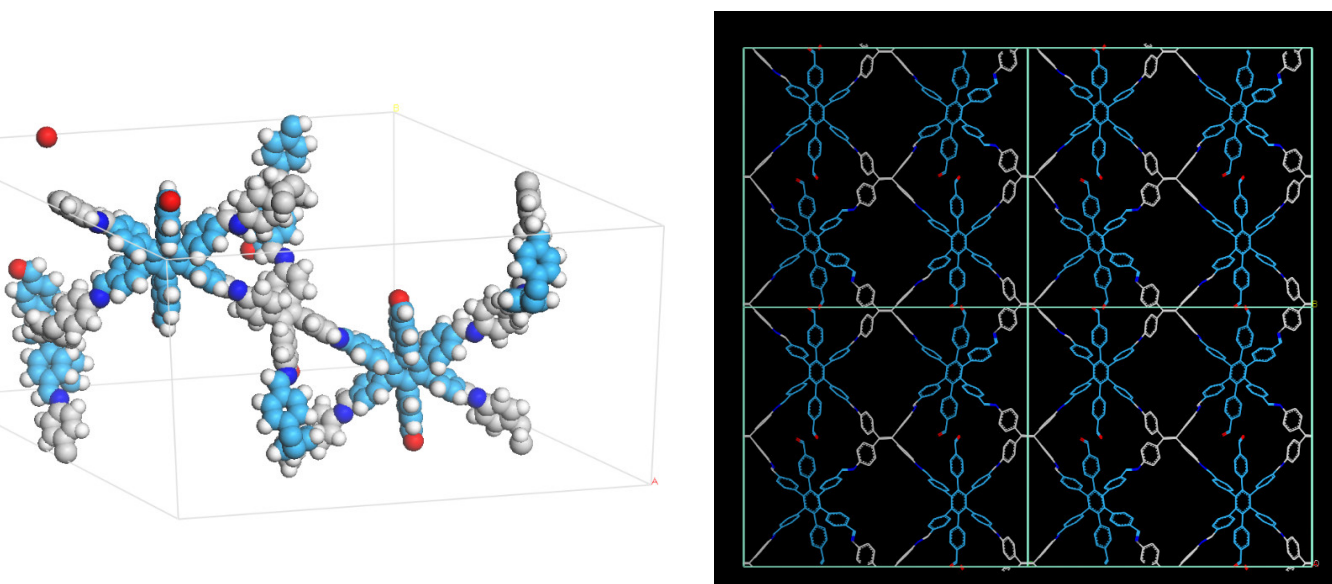

Figure S22. Structural illustration for possible 3D lvt-a topology built from HFB (tetratopic) and TPE-NH2. 

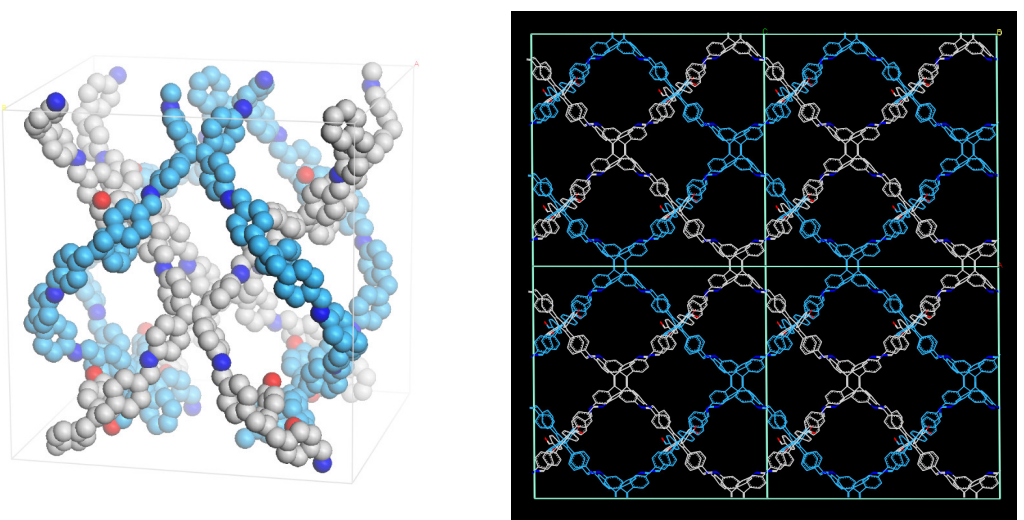

Figure S23. Structural illustration for possible 3D lvt-a (2-fold interpenetrated) topology built from HFB (tetratopic) and TPE-NH2.

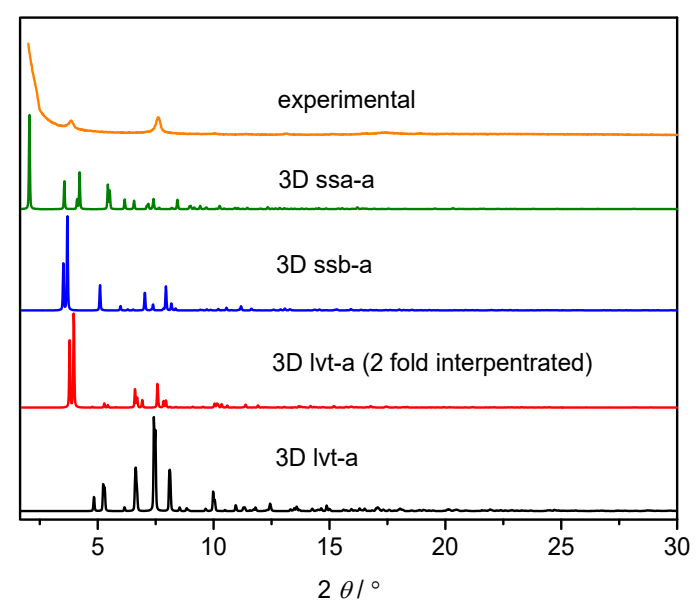

Figure S24. PXRD patterns $(\mathrm{Cu} \mathrm{K} \alpha)$ for possible partially connected topologies of 3D structures constructed from HFB (tetratopic) and TPE-NH2.

2) $\mathrm{C}_{3}+\mathrm{C}_{4} /$ tetratopic:

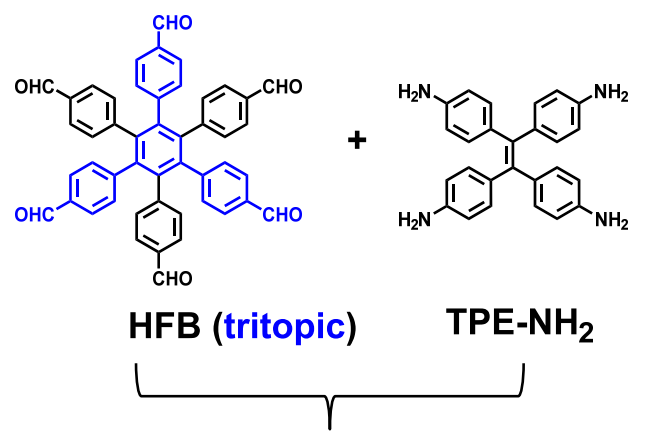

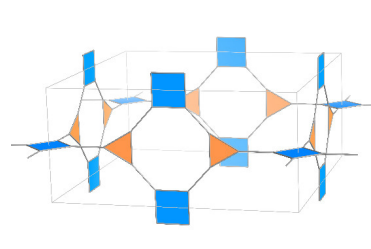

ffc

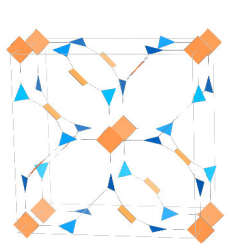

fjh-a

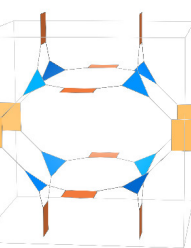

pto-a

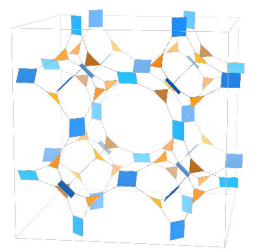

tbo-a

Figure S25. Possible partially connected topologies of 3D structures constructed from HFB (tritopic) and TPE-NH2. 

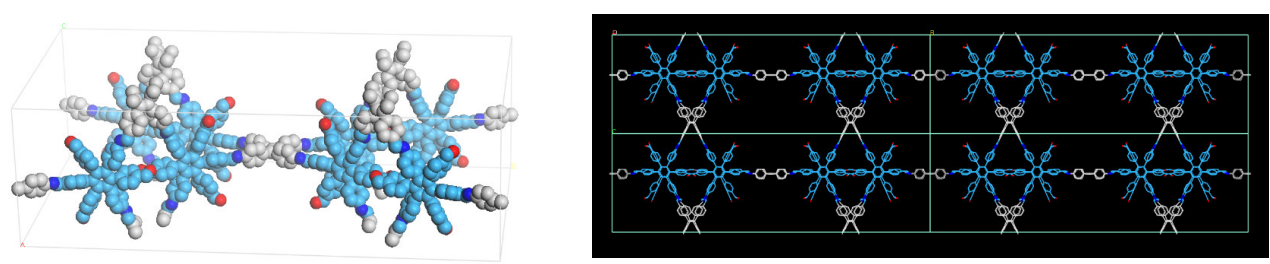

Figure S26. Structural illustration for possible 3D ffc topology built from HFB (tritopic) and TPE-NH2.
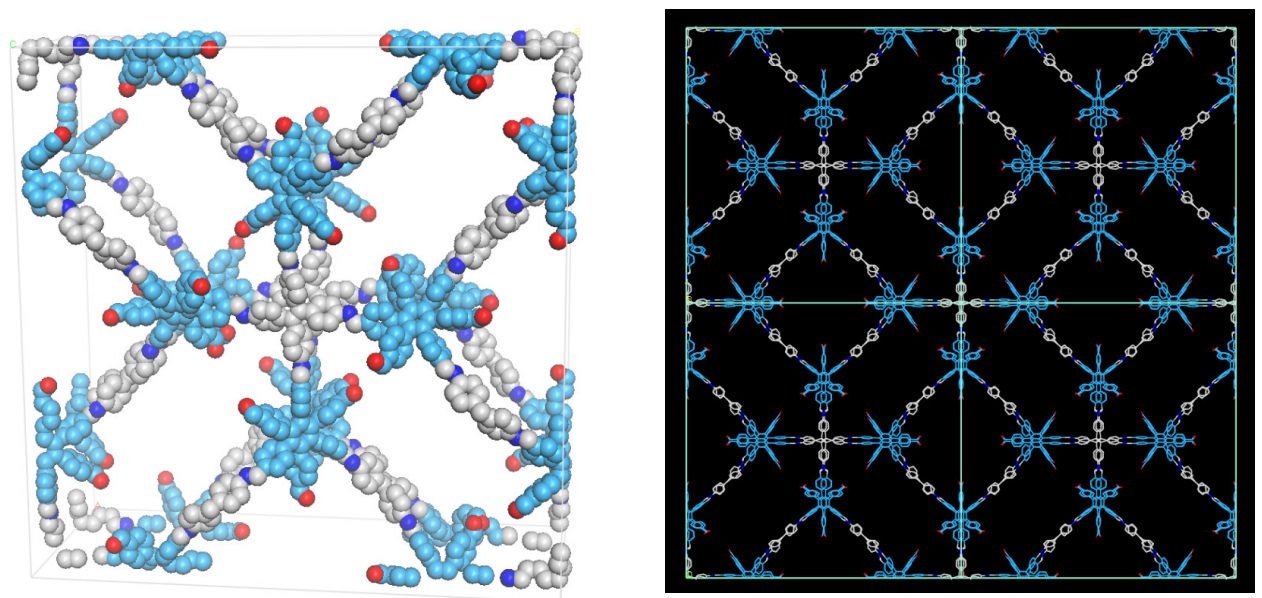

Figure S27. Structural illustration for possible 3D fjh-a topology built from HFB (tritopic) and TPE-NH2.
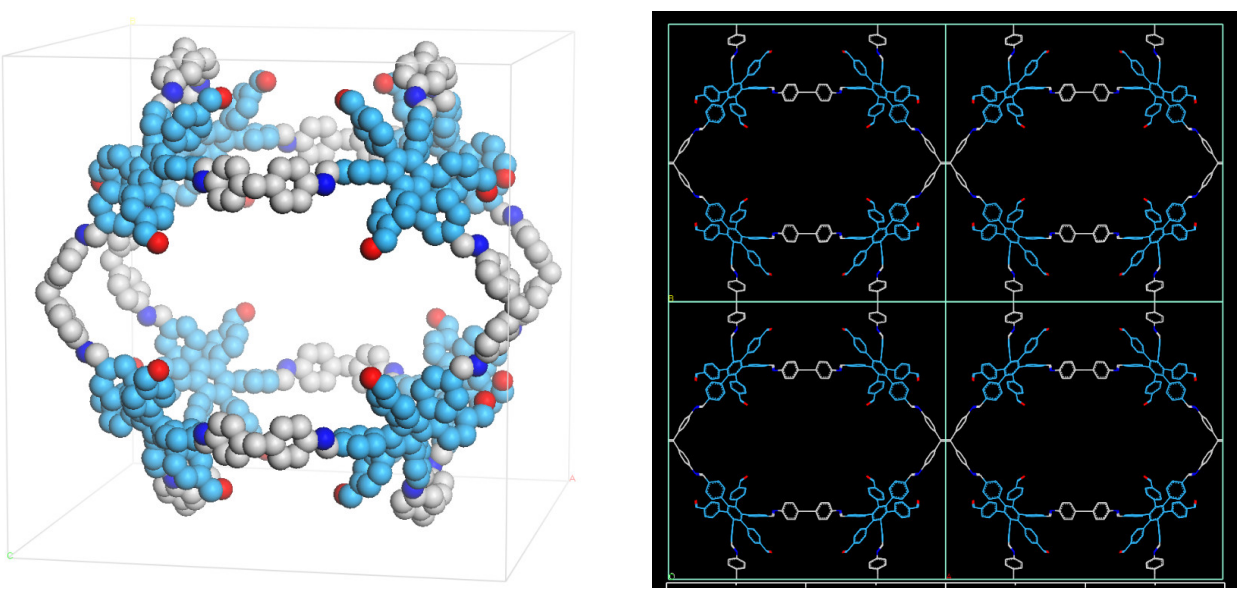

Figure S28. Structural illustration for possible 3D pto-a topology built from HFB (tritopic) and TPE-NH2. 

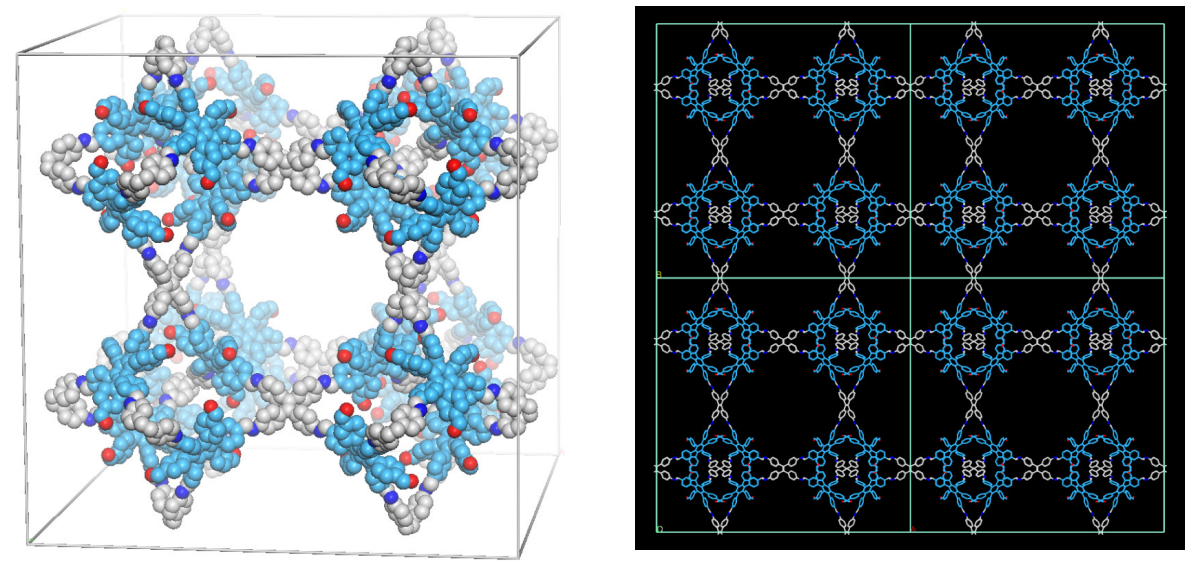

Figure S29. Structural illustration for possible 3D tbo-a topology built from HFB (tritopic) and TPE-NH2.

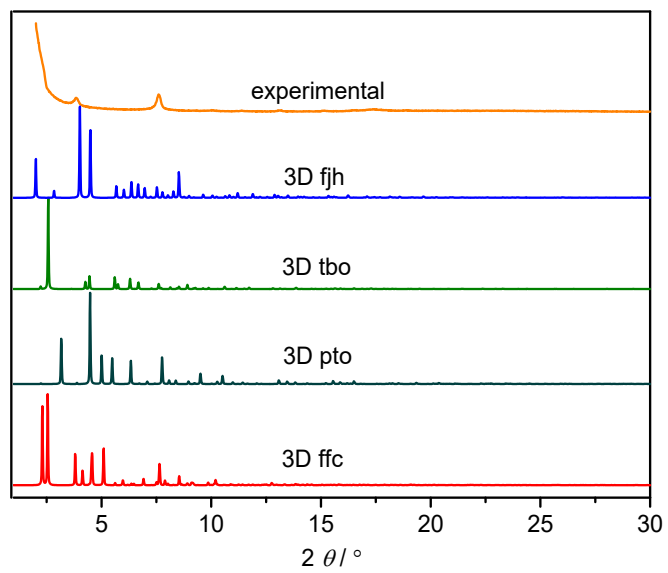

Figure S30. PXRD patterns $(\mathrm{Cu} \mathrm{K} \alpha)$ for possible partially connected topologies of 3D structures constructed from HFB (tritopic) and TPE-NH2.

3) $\mathrm{C}_{2}+\mathrm{C}_{4} /$ tetratopic or $\mathrm{C}_{4} /$ tetratopic $+\mathrm{C}_{2}$ :

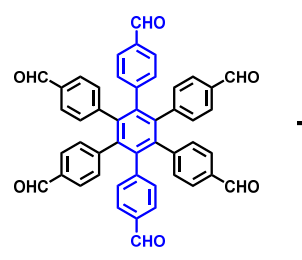

HFB (ditopic)

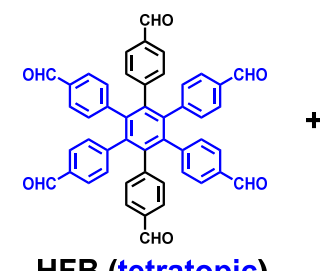

HFB (tetratopic)

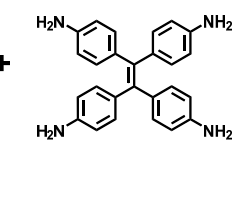

TPE-NH

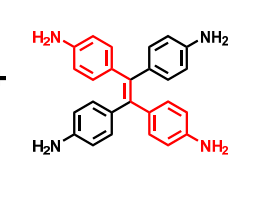

TPE-NH (ditopic) $^{2}$

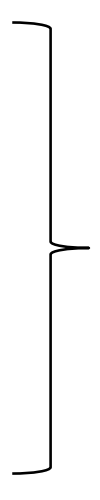

kgm

sql
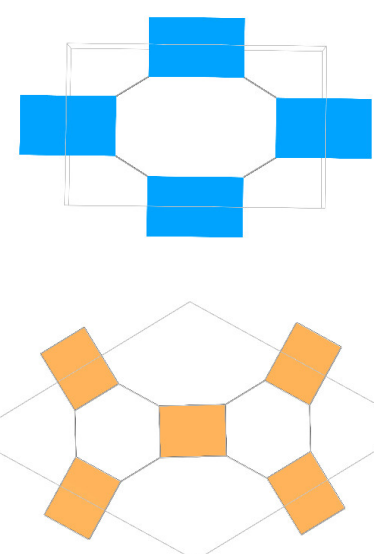

Figure S31. Possible partially connected topologies of 2D structures constructed from HFB 
and TPE-NH2.

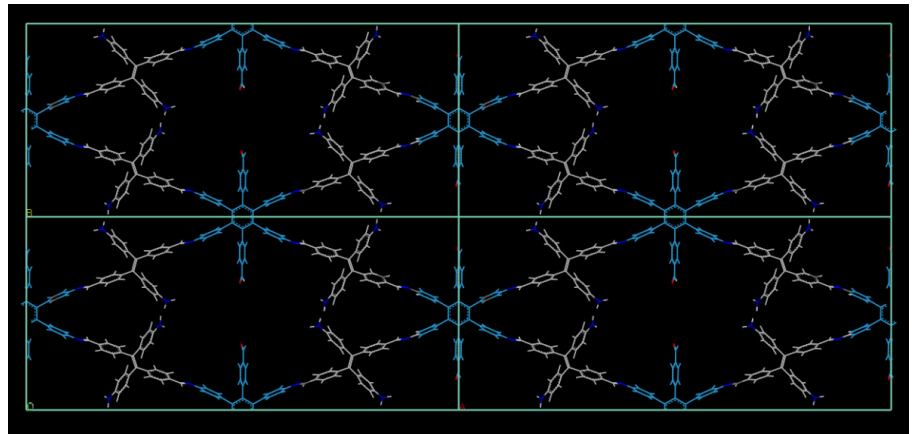

Figure S32. Structural illustration for possible 2D sql topology built from HFB (tetratopic) and TPE-NH2 (ditopic).

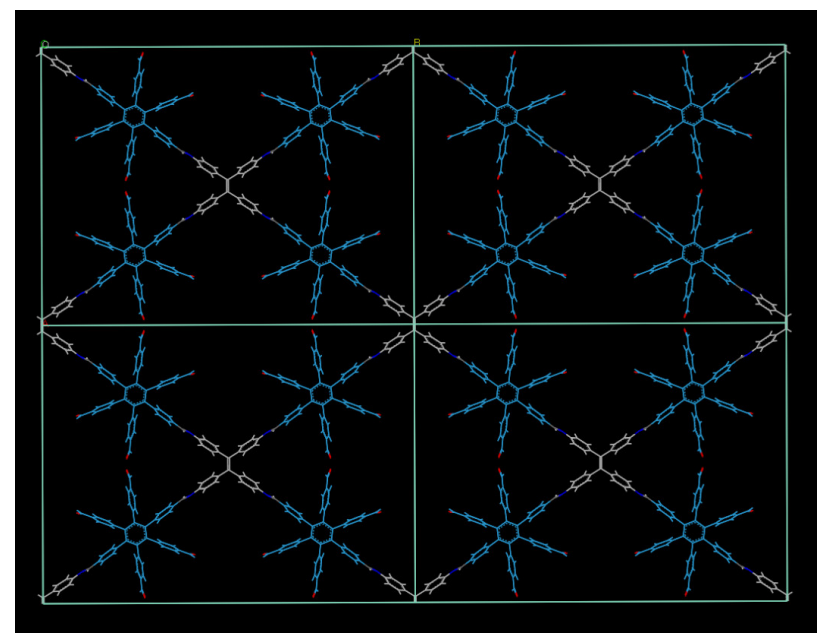

Figure S33. Structural illustration for possible 2D sql topology built from HFB (ditopic) and TPE-NH2.

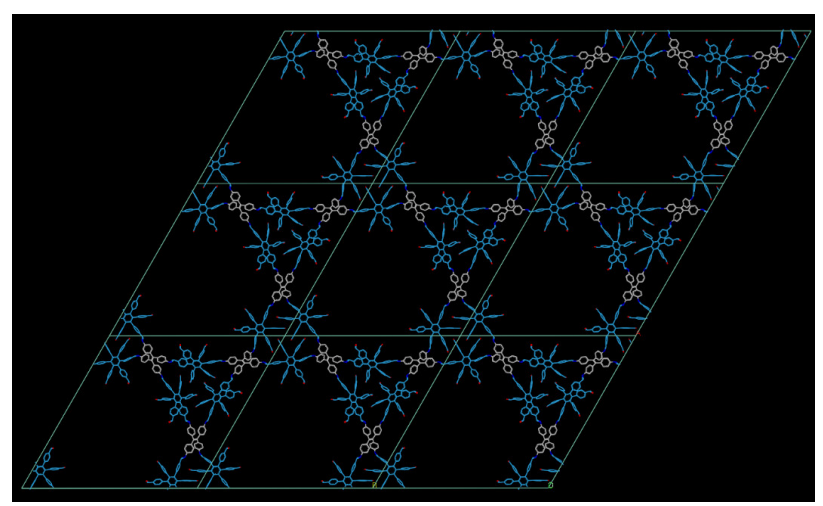

Figure S34. Structural illustration for possible 2D kgm topology built from HFB (ditopic) and TPE-NH2. 


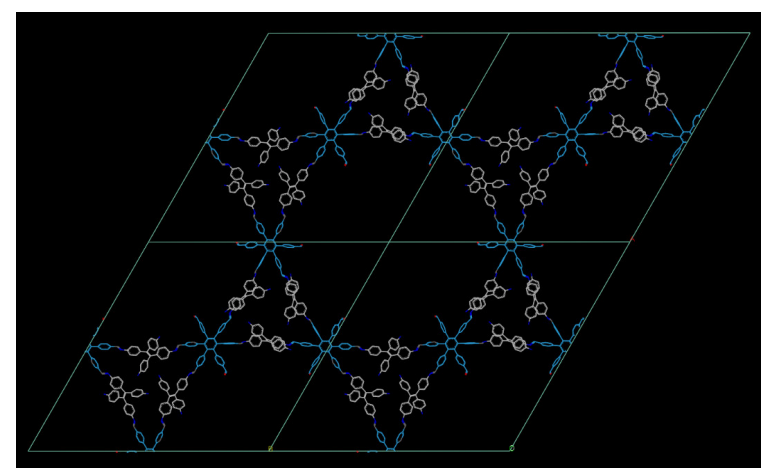

Figure S35. Structural illustration for possible 2D kgm topology built from HFB (tetratopic) and TPE-NH $\mathbf{N H}_{2}$ (ditopic).

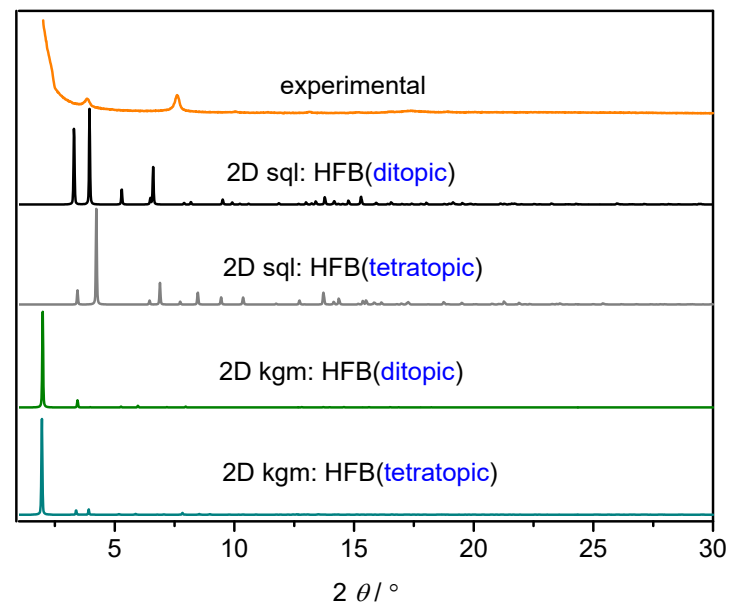

Figure S36. PXRD patterns $(\mathrm{Cu} \mathrm{K} \alpha)$ for possible partially connected topologies of 2D structures constructed from HFB and TPE-NH2.

4) $\mathrm{C}_{4} /$ tetratopic $+\mathrm{T}_{\mathrm{d}}$ :

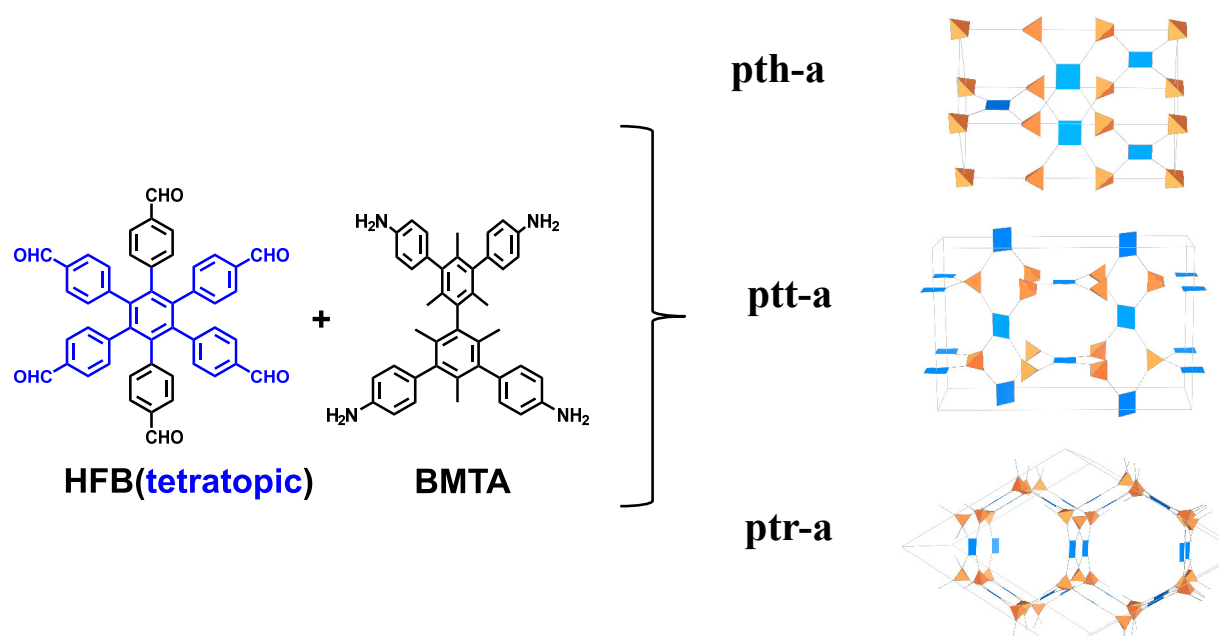

Figure S37. Possible partially connected topologies of 3D structures constructed from HFB (tetratopic) and BMTA. 

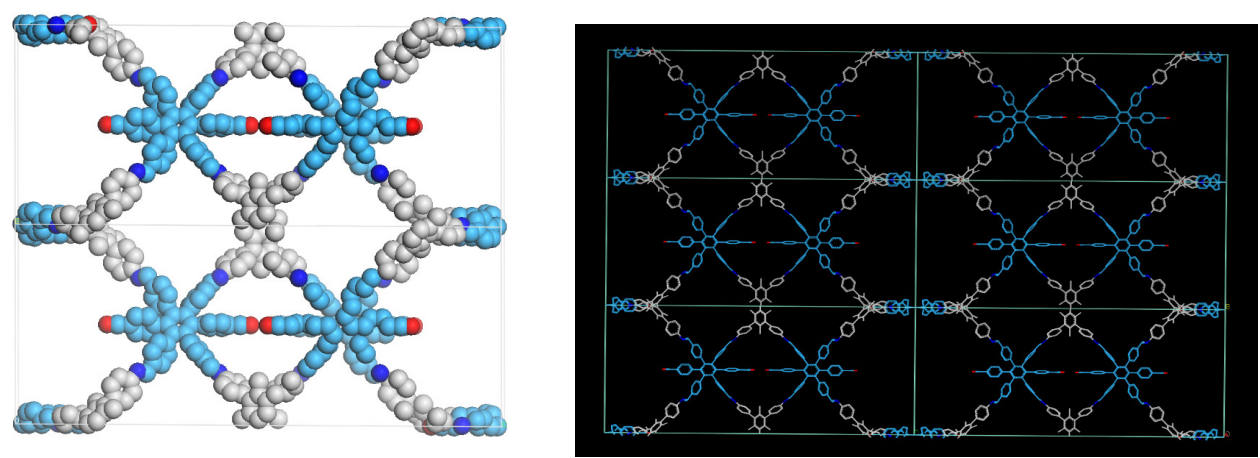

Figure S38. Structural illustration for possible 3D pth-a topology built from HFB (tetratopic) and BMTA.
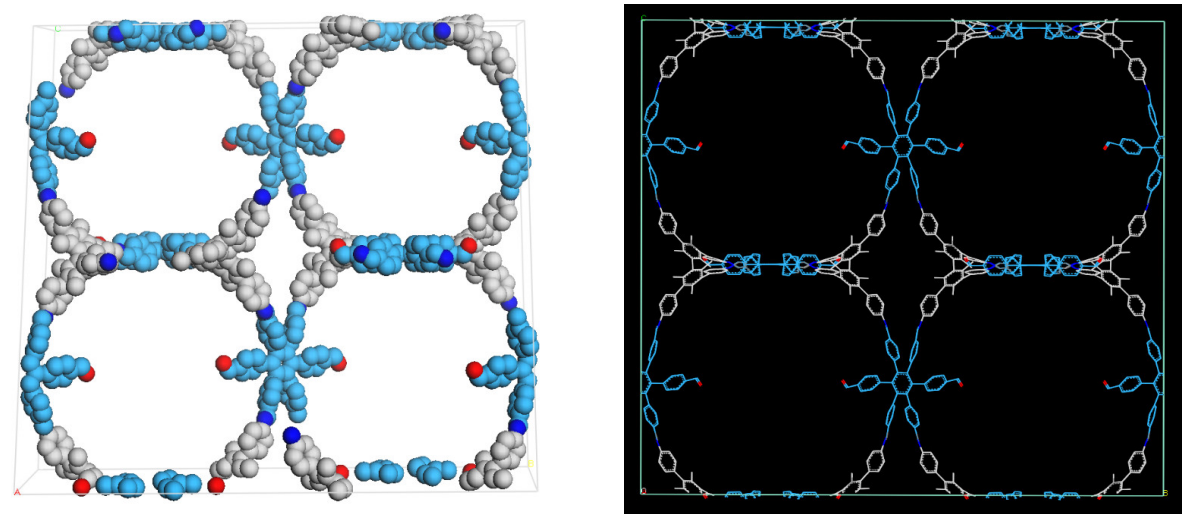

Figure S39. Structural illustration for possible 3D ptt-a topology built from HFB (tetratopic) and BMTA.
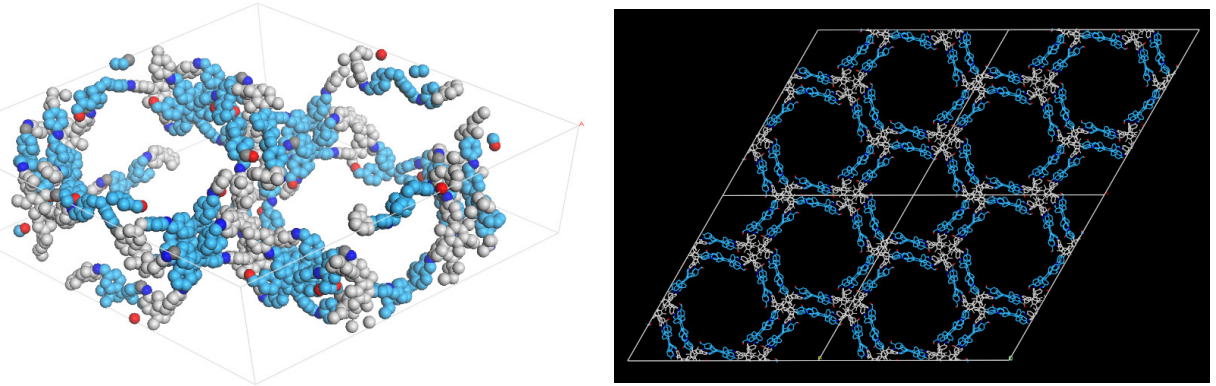

Figure S40. Structural illustration for possible 3D ptr-a topology built from HFB (tetratopic) and BMTA. 


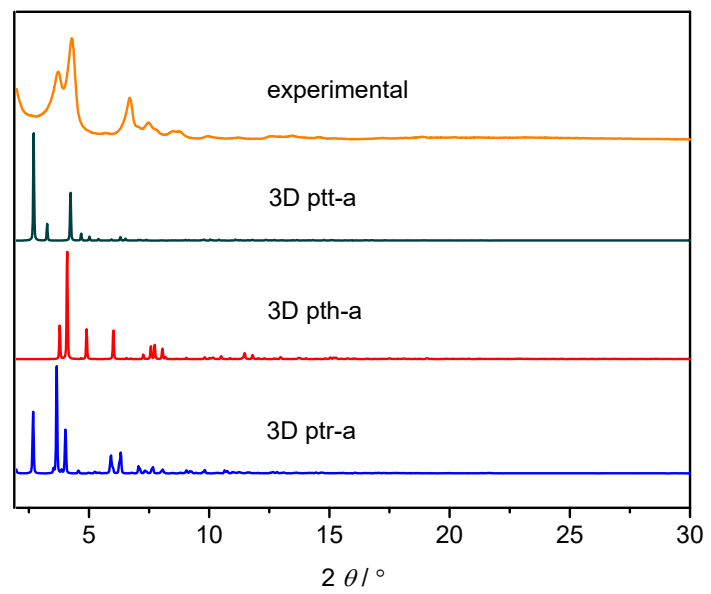

Figure S41. PXRD patterns $(\mathrm{Cu} \mathrm{K} \alpha)$ for possible partially connected topologies of 3D structures constructed from HFB (tetratopic) and BMTA.

5) $\mathrm{C}_{3}+\mathrm{T}_{\mathrm{d}}$ :

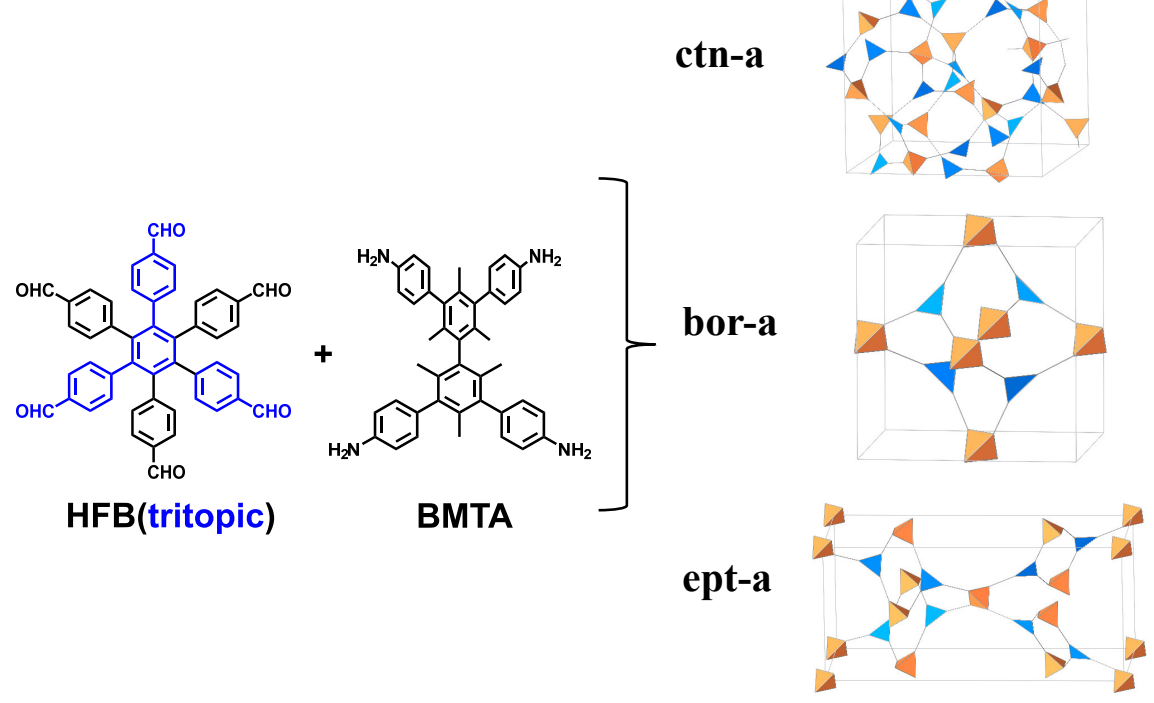

Figure S42. Possible partially connected topologies of 3D structures constructed from HFB (tritopic) and BMTA.
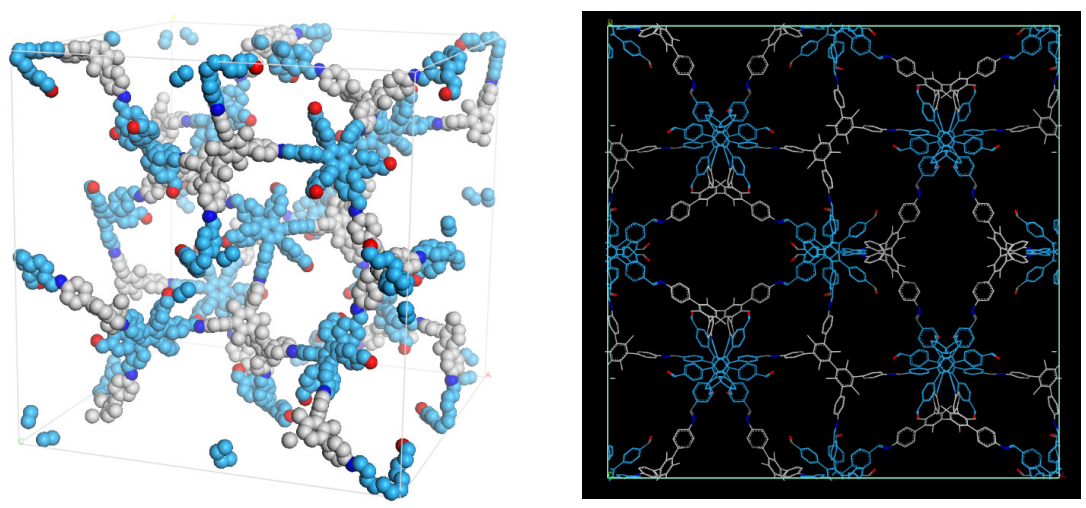

Figure S43. Structural illustration for possible 3D ctn-a topology built from HFB (tritopic) and BMTA. 

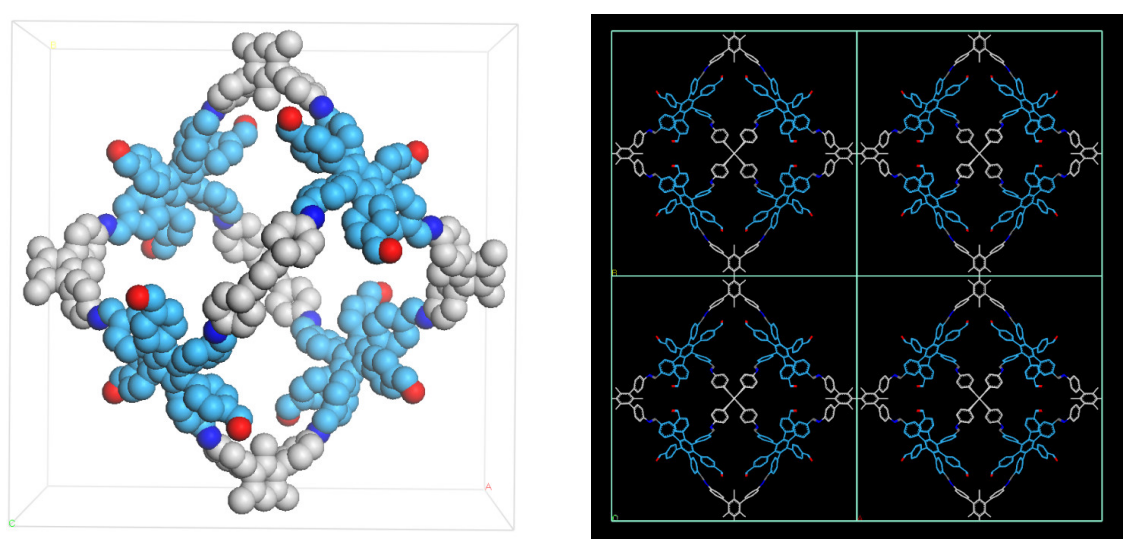

Figure S44. Structural illustration for possible 3D bor-a topology built from HFB (tritopic) and BMTA.
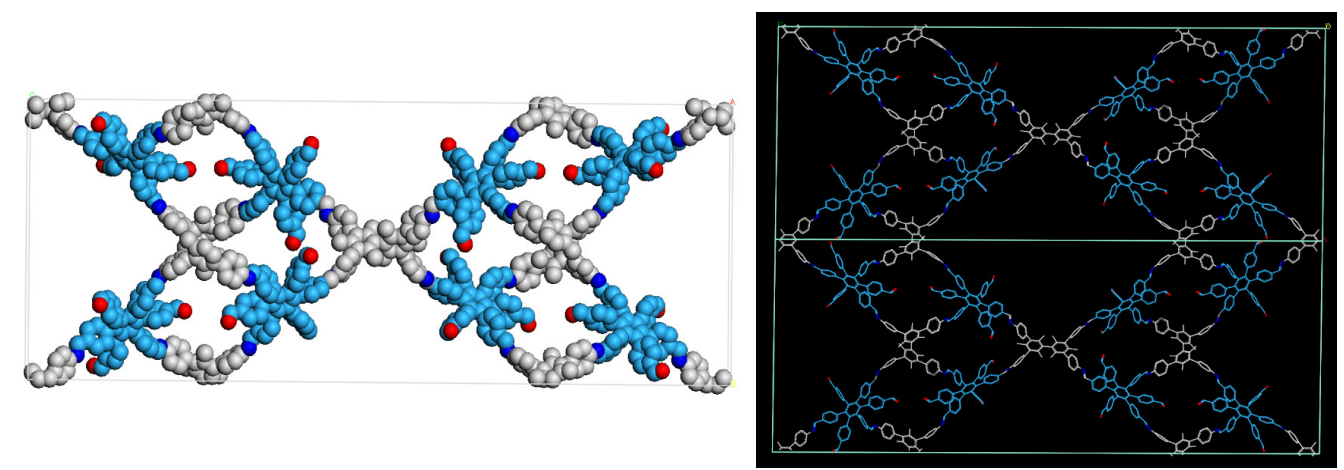

Figure S45. Structural illustration for possible 3D ept-a topology built from HFB (tritopic) and BMTA.

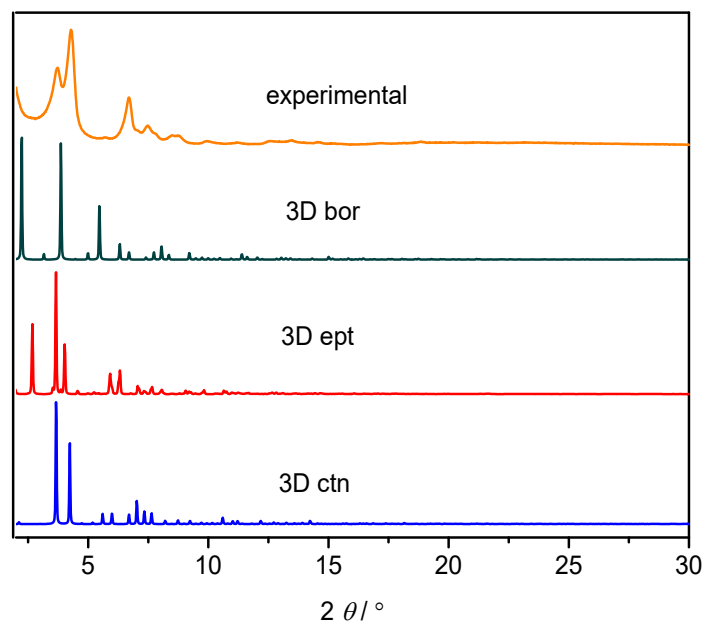

Figure S46. PXRD patterns $(\mathrm{Cu} \mathrm{K} \alpha)$ for possible partially connected topologies of 3D structures constructed from HFB (tritopic) and BMTA. 


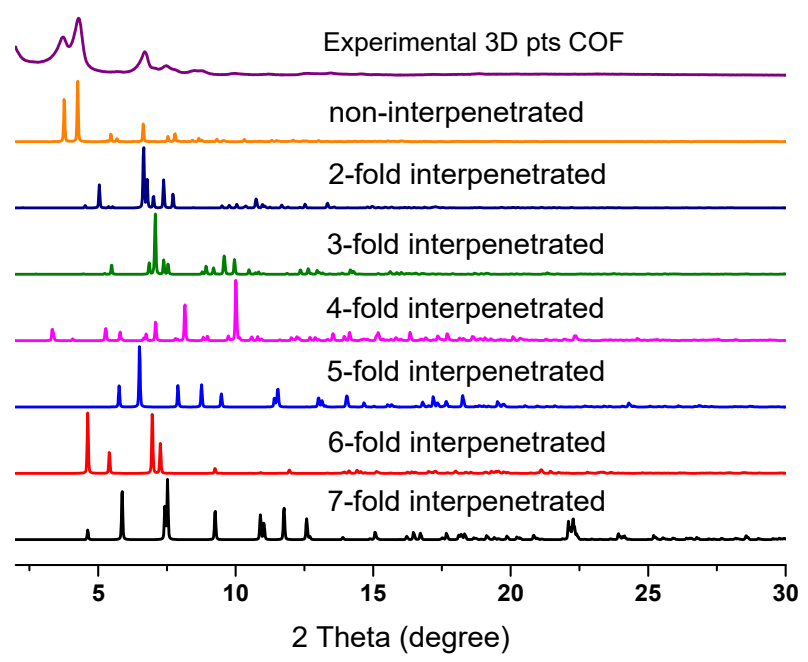

Figure S47. PXRD patterns $(\mathrm{Cu} \mathrm{K \alpha})$ for different fold interpenetrated pts structures constructed from HFB (tetratopic) and BMTA.

(a)

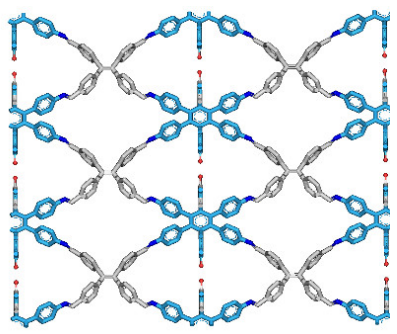

(b)

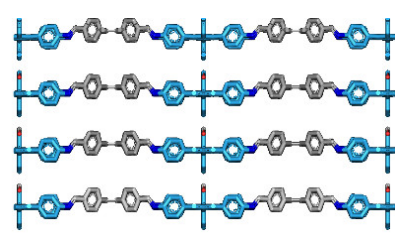

(c)

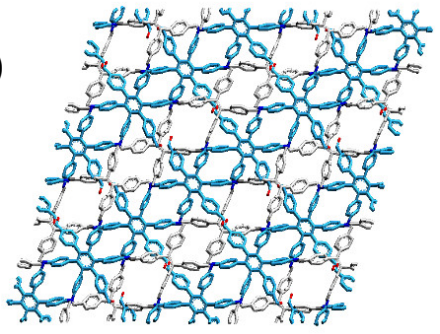

(d)

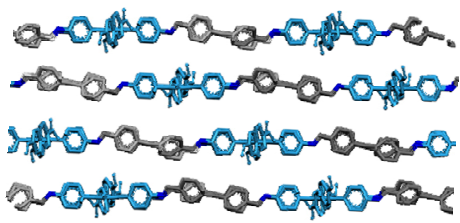

(e)

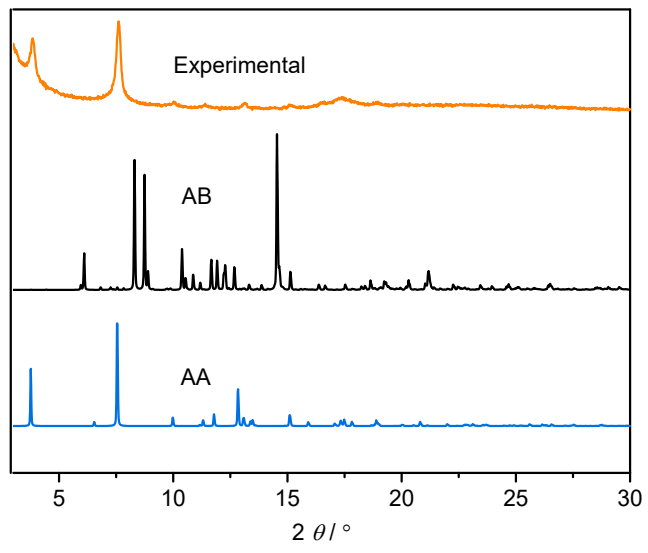

Figure S48. (a) Top and (b) side views of ball and stick structural model for inclined 2D sql COF (AA). (c) Top and (d) side views of ball and stick structural model for inclined 2D sql COF (AB). HFB in blue, TPE-NH $\mathbf{N H}_{2}$ in grey, $\mathrm{O}$ atoms in red. (e) PXRD patterns $(\mathrm{Cu} \mathrm{K} \alpha)$ for inclined 2D sql COF (AA) and 2D sql COF (AB) model. 
Table S2. Pawley and Rietveld refinement results for COFs.

\begin{tabular}{|c|c|c|c|}
\hline 2D sql COF & Unit cell & Rwp (\%) & $\mathrm{Rp}(\%)$ \\
\hline Pawley & $\begin{array}{c}\mathrm{a}=13.51 \AA ; \mathrm{b}=23.47 \AA ; \\
\mathrm{c}=7.92 \AA \\
\alpha=\gamma=90^{\circ}, \beta=91.16^{\circ}\end{array}$ & $4.22 \%$ & $3.28 \%$ \\
\hline Rietveld & $\begin{array}{c}\mathrm{a}=13.60 \AA ; \mathrm{b}=23.50 \AA ; \mathrm{c} \\
=7.83 \AA \\
\alpha=\gamma=90^{\circ}, \beta=90.56^{\circ}\end{array}$ & $4.80 \%$ & $3.75 \%$ \\
\hline 3D pts COF & Unit cell & Rwp (\%) & $\mathrm{Rp}(\%)$ \\
\hline Pawley & $\begin{array}{c}\mathrm{a}=\mathrm{b}=23.46 \AA ; \mathrm{c}=44.66 \\
\AA \\
\alpha=\beta=\gamma=90^{\circ}\end{array}$ & $4.38 \%$, & $3.29 \%$ \\
\hline Rietveld & $\begin{array}{c}\mathrm{a}=\mathrm{b}=24.17 \AA ; \mathrm{c}=43.17 \\
\AA \\
\alpha=\beta=\gamma=90^{\circ}\end{array}$ & $5.40 \%$ & $4.02 \%$ \\
\hline COF-TPB & Unit cell & Rwp (\%) & $\mathrm{Rp}(\%)$ \\
\hline Pawley & $\begin{array}{c}a=45.87 \AA ; b=29.51 \AA \\
c=6.43 \AA \\
\alpha=\gamma=90^{\circ}, \beta=85.17^{\circ}\end{array}$ & $4.12 \%$ & $3.28 \%$ \\
\hline Rietveld & $\begin{array}{c}a=46.62 \AA ; b=29.74 \AA \\
c=6.37 \AA \\
\alpha=\gamma=90^{\circ}, \beta=81.07^{\circ}\end{array}$ & $5.32 \%$ & $4.16 \%$ \\
\hline
\end{tabular}

\section{3. 3 Control experiments}

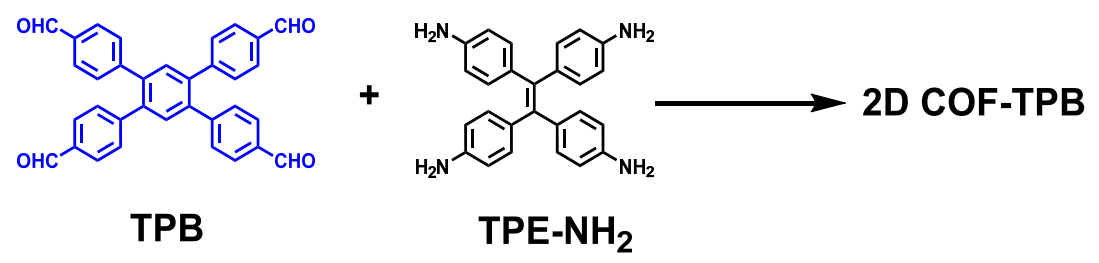




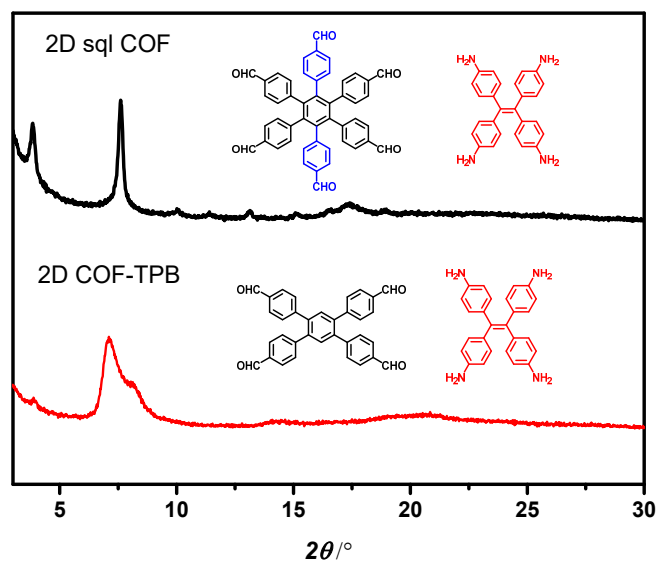

Figure S49. PXRD patterns for COF-TPB, showing the same XRD pattern as that of 2D sql COF.

(a)

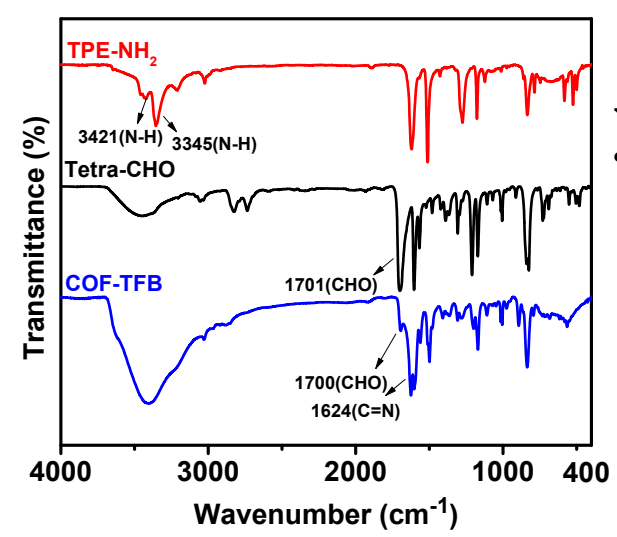

(b)

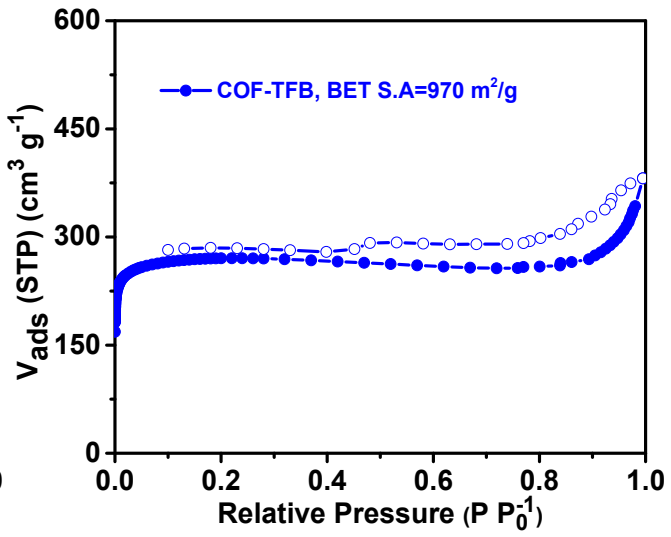

(c)

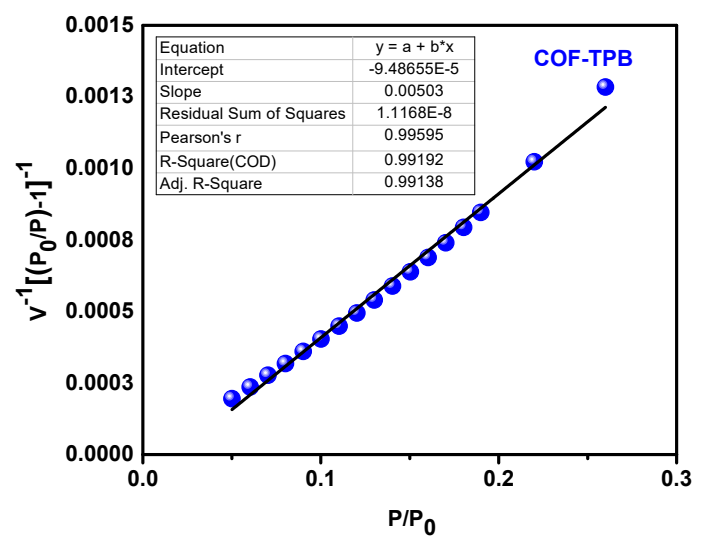

Figure S50. (a) FT-IR spectrum and (b) Nitrogen sorption curves, and (c) BET surface areas for COF-TPB. 
(a)

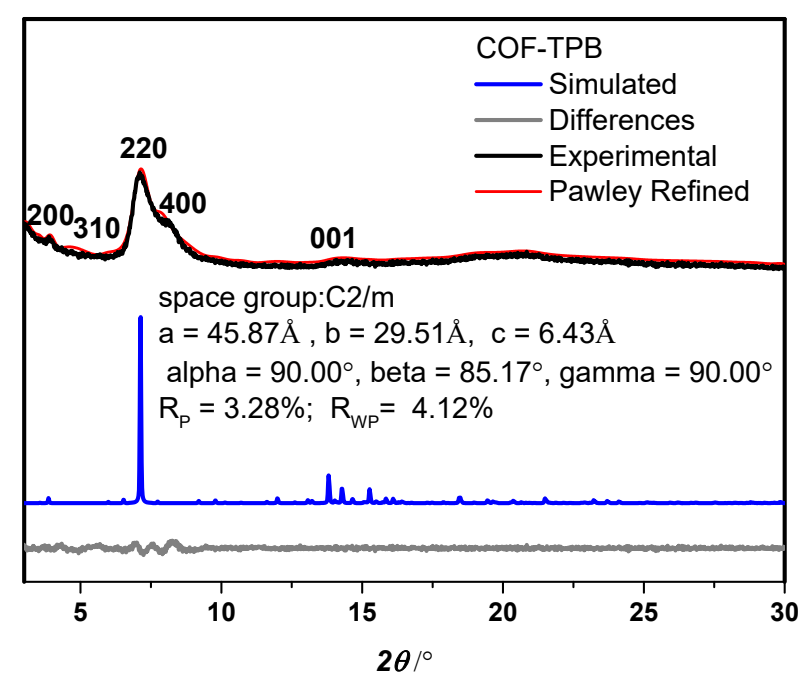

(b)
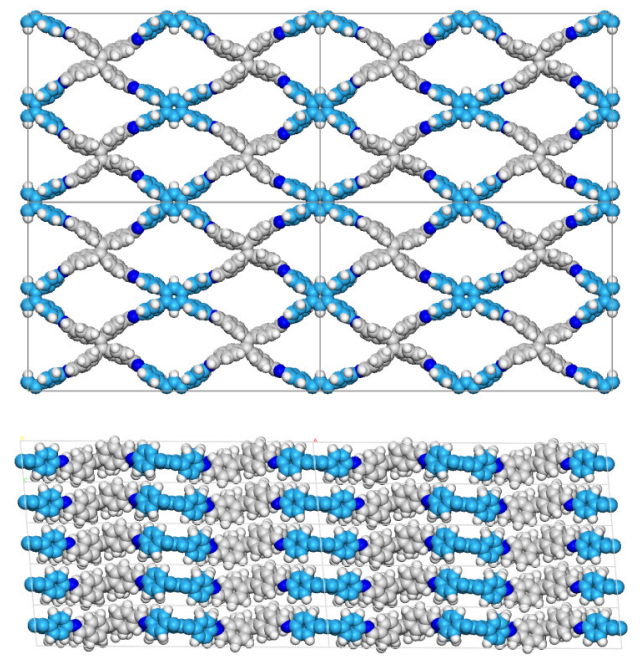

Figure S51. (a) PXRD pattern and refinement fitting for COF-TPB. (b) Top and side views of structural model for COF-TPB.

(a)
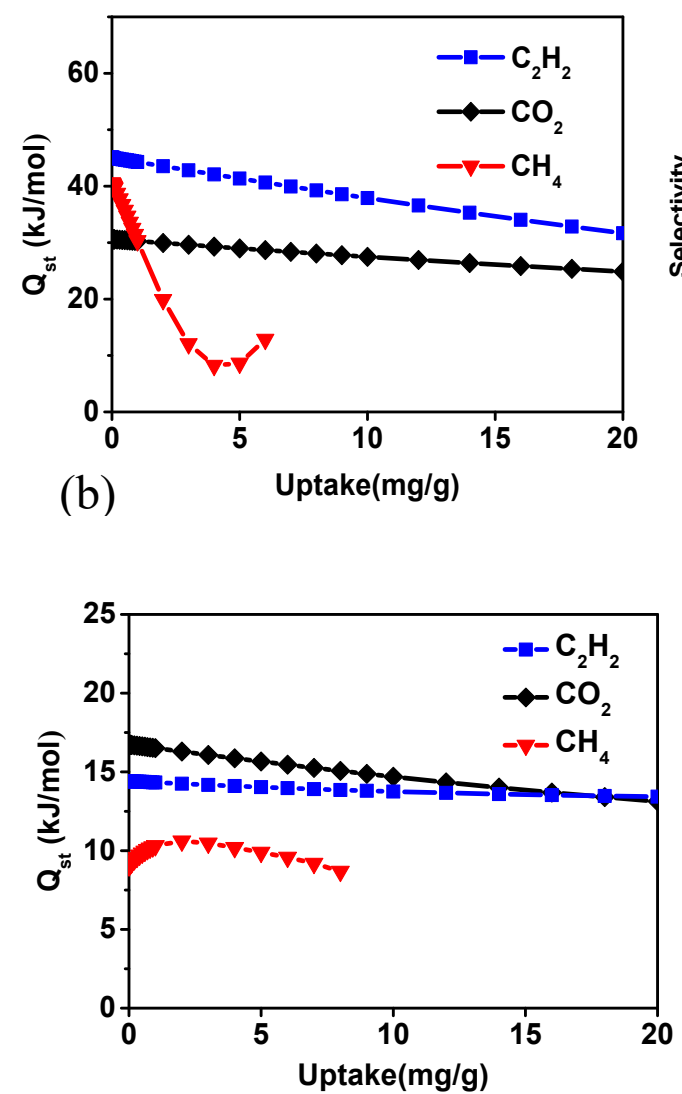

(c)

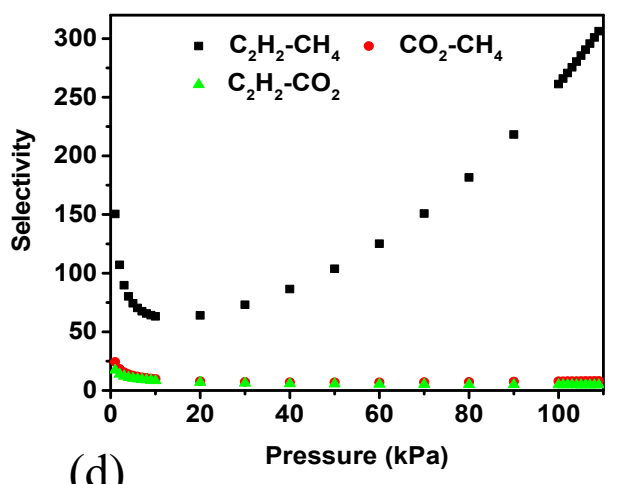

(d)

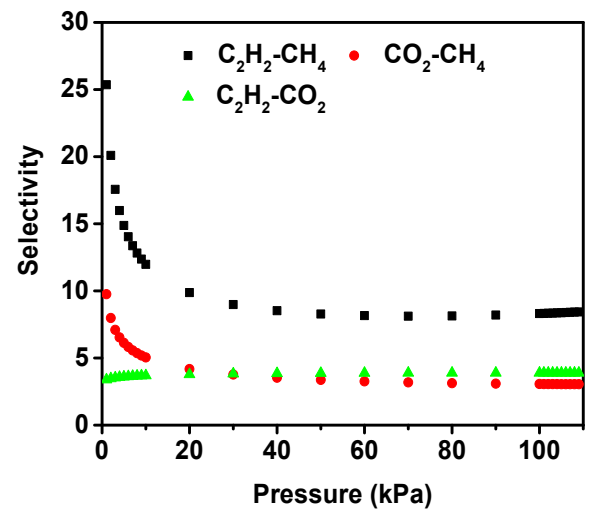

Figure S52. Coverage-dependent adsorption enthalpies of $\mathrm{C}_{2} \mathrm{H}_{2}, \mathrm{CO}_{2}$, and $\mathrm{CH}_{4}$ at $298 \mathrm{~K}$ for (a) 2D sql COF and (b) 3D pts COF. Selectivities of equimolar binary $\mathrm{C}_{2} \mathrm{H}_{2} / \mathrm{CH}_{4}, \mathrm{CO}_{2} / \mathrm{CH}_{4}$, 
and $\mathrm{C}_{2} \mathrm{H}_{2} / \mathrm{CO}_{2}$ at $298 \mathrm{~K}$ for (c) 2D sql COF and (d) 3D pts COF.

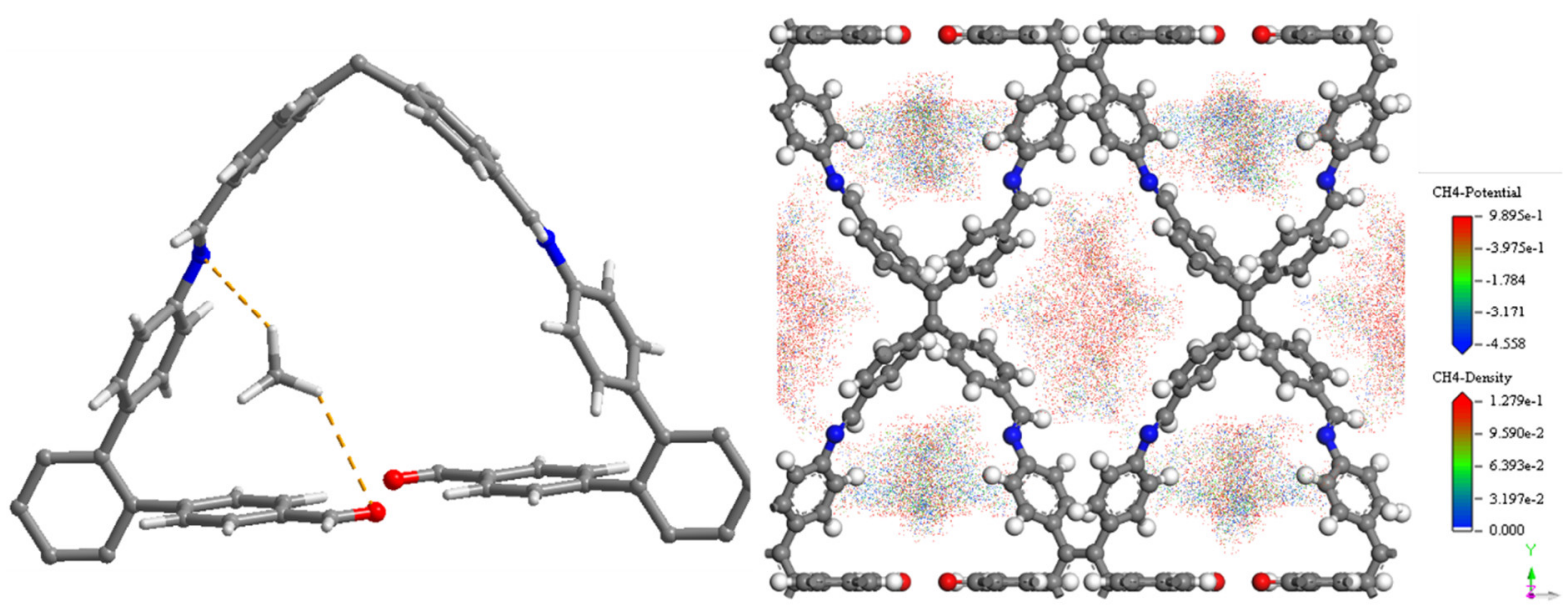

Figure S53. GCMC simulation results displaying the preferential $\mathrm{CH}_{4}$ binding site and distribution of $\mathrm{CH}_{4}$ adsorbed in $2 \mathrm{D}$ sql COFs.

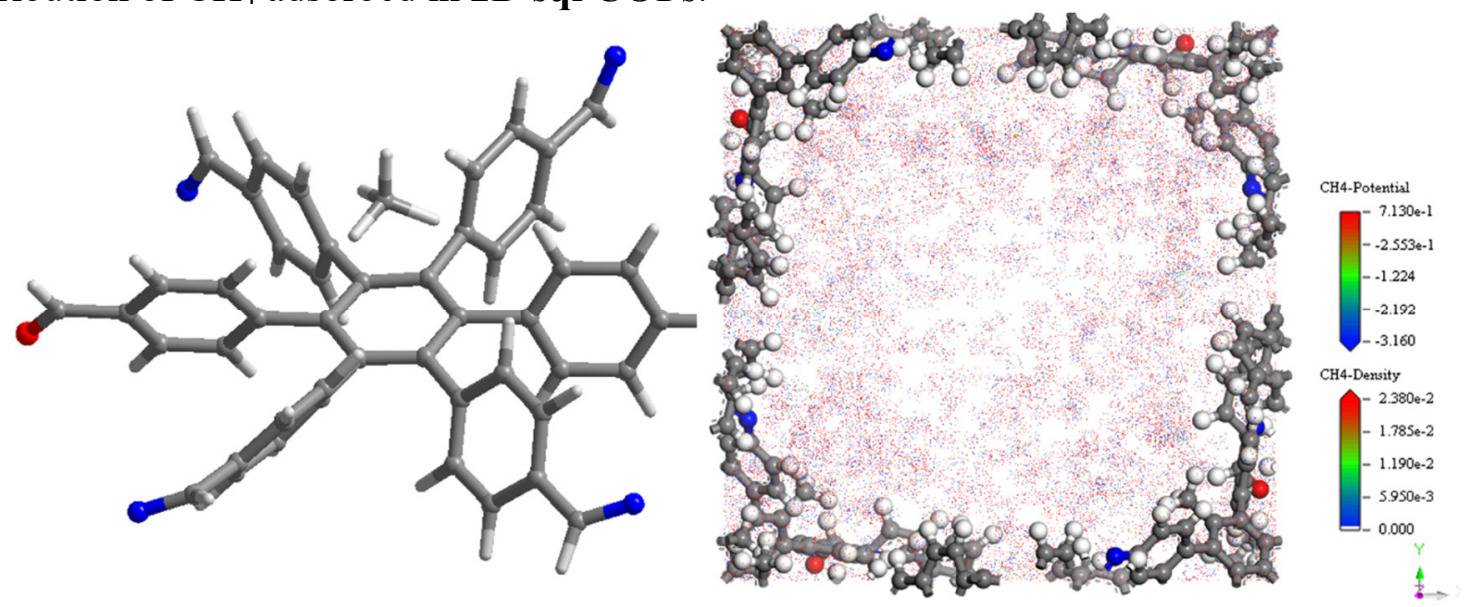

Figure S54. GCMC simulation results displaying the preferential $\mathrm{CH}_{4}$ binding site and distribution of $\mathrm{CH}_{4}$ adsorbed in 3D pts COFs.

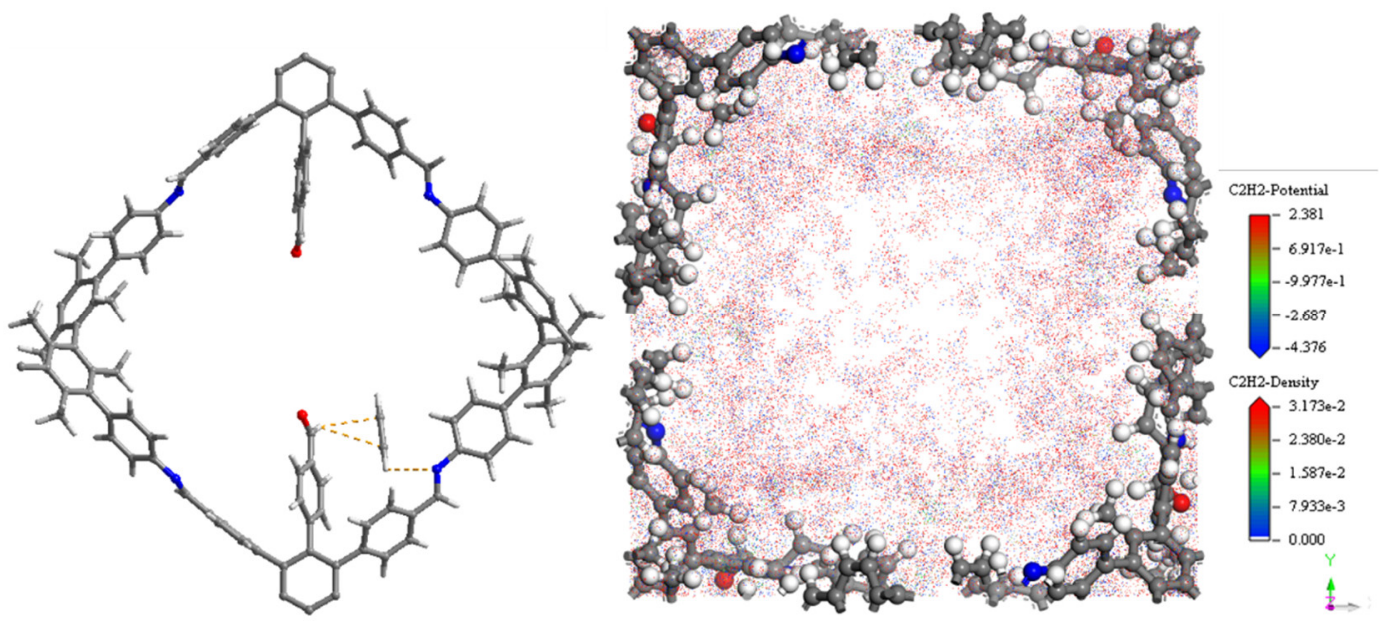

Figure S55. GCMC simulation results displaying the preferential $\mathrm{C}_{2} \mathrm{H}_{2}$ binding site and distribution of $\mathrm{C}_{2} \mathrm{H}_{2}$ adsorbed in 3D pts COFs. 


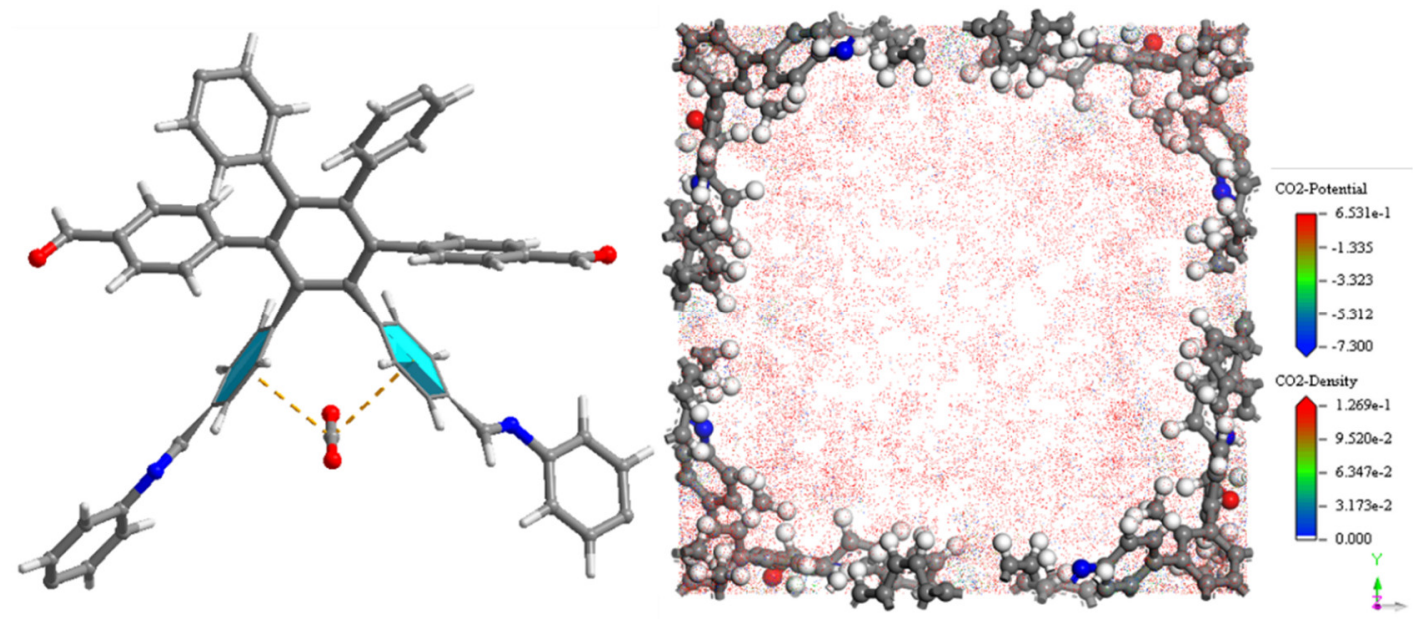

Figure S56. GCMC simulation results displaying the preferential $\mathrm{CO}_{2}$ binding site and distribution of $\mathrm{CO}_{2}$ adsorbed in 3D pts COFs.

\section{References}

(S1) Materials Studio; Accelrys: San Diego.

(S2) http://www.rcsr.net/

(S3) http://www.ba.ic.cnr.it/softwareic/expo/

(S4) http://www.jp-minerals.org/vesta/en/

(S5) http://www.zeoplusplus.org/

(S6) Bing Zhang, Haiyan Mao, Roc Matheu, Jeffrey A. Reimer, Sultan A. Alshmimri, Saeed Alshihri, and Omar M. Yaghi. J. Am. Chem. Soc 2019, 141, 11420.

(S7) Chao Gao, Jian Li, Sheng Yin, Junliang Sun, and Cheng Wang. J. Am. Chem. Soc 2020, 142, 3718 . 